\title{
LinkPred: A High Performance Library for Link Prediction in Complex Networks
}

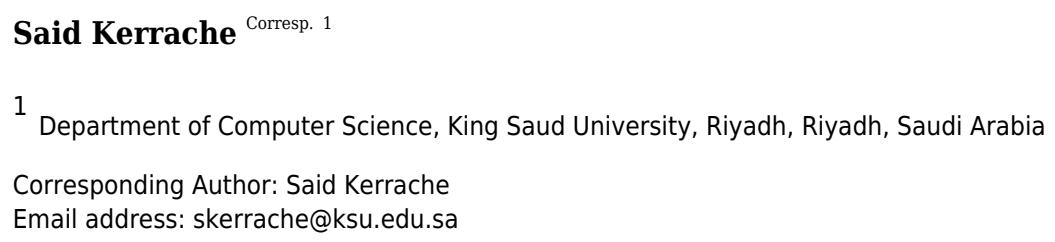

The problem of determining the likelihood of the existence of a link between two nodes in a network is called link prediction. This is made possible thanks to the existence of a topological structure in most real-life networks. In other words, the topologies of networked systems such as the World Wide Web, the Internet, metabolic networks, and human society are far from random, which implies that partial observations of these networks can be used to infer information about undiscovered interactions. Significant research efforts have been invested into the development of link prediction algorithms, and some researchers have made the implementation of their methods available to the research community. These implementations, however, are often written in different languages and use different modalities of interaction with the user, which hinders their effective use. This paper introduces LinkPred, a high-performance parallel and distributed link prediction library that includes the implementation of the major link prediction algorithms available in the literature. The library can handle networks with up to millions of nodes and edges and offers a unified interface that facilitates the use and comparison of link prediction algorithms by researchers as well as practitioners. 


\title{
LinkPred: A High Performance Library for Link Prediction in Complex Networks
}

\section{Said Kerrache}

\author{
Department of Computer Science, King Saud University, Riyadh 11543, KSA
}

Corresponding author:

Said Kerrache

Email address: skerrache@ksu.edu.sa

\begin{abstract}
The problem of determining the likelihood of the existence of a link between two nodes in a network is called link prediction. This is made possible thanks to the existence of a topological structure in most real-life networks. In other words, the topologies of networked systems such as the World Wide Web, the Internet, metabolic networks, and human society are far from random, which implies that partial observations of these networks can be used to infer information about undiscovered interactions. Significant research efforts have been invested into the development of link prediction algorithms, and some researchers have made the implementation of their methods available to the research community. These implementations, however, are often written in different languages and use different modalities of interaction with the user, which hinders their effective use. This paper introduces LinkPred, a highperformance parallel and distributed link prediction library that includes the implementation of the major link prediction algorithms available in the literature. The library can handle networks with up to millions of nodes and edges and offers a unified interface that facilitates the use and comparison of link prediction algorithms by researchers as well as practitioners.
\end{abstract}

\section{INTRODUCTION}

The field of complex networks, and more generally that of network science, aims at studying networked systems, that is, systems composed of a large number of interacting components (Albert and Barabási, 2002). Under this umbrella fall many seemingly disparate networks, but which share common underlying topological properties that constitute a fertile ground for analyzing and ultimately understanding these systems. Networks of interest can be social, biological, informational, or technological. Link prediction is the task of identifying links missing from a network (Lü and Zhou, 2011; Martínez et al., 2016; Guimerà and Sales-Pardo, 2009; Al Hasan et al., 2006; Guimerà and Sales-Pardo, 2009; Clauset et al., 2008; Lü and Zhou, 2011; Cannistraci et al., 2013; Daminelli et al., 2015; Al Hasan et al., 2006; Wang et al., 2007; Zhang et al., 2020; Beigi et al., 2020; Sajadmanesh et al., 2019; Makarov et al., 2019), a problem with important applications, such as the reconstruction of networks from partial observations (Guimerà and Sales-Pardo, 2009), recommendation of items in online shops and friends in social networks (Al Hasan et al., 2006), and the prediction of interactions in biological networks (Clauset et al., 2008).

This paper introduces LinkPred, a C++ high-performance link prediction library that includes the implementation of the major link prediction algorithms available in the literature by development from scratch and wrapping or translating existing implementations. The library is designed with three guiding principles, ease of use, extensibility, and efficiency. To facilitate its use, LinkPred borrows heavily from the STL design to offer an elegant and powerful interface. C++ users with minimum experience using STL will find the library's programming and usage style to be very familiar. Moreover, the use of templates allows for greater flexibility when using LinkPred and allows for integration within various contexts. The library contains bindings to Java and Python, providing access to its main functionalities through easy-to-use classes. LinkPred is aimed not only at practitioners but also at researchers in the field. It is designed to allow developers of new link prediction algorithms to easily integrate their code into the library and evaluate its performance. Efficiency-wise, the data structures used and implemented in LinkPred are all chosen and designed to achieve high performance. Additionally, most code in LinkPred is 
parallelized using OpenMp, which allows taking advantage of shared memory architectures. Furthermore, a significant portion of the implemented predictors supports distributed processing using MPI, allowing the library to handle very large networks with up to hundreds of thousands to millions of nodes.

In the rest of this paper, an overview of related software packages is presented first, followed by a description of the library's architecture and main functionalities. Example use cases with fully working code samples are presented next. The paper is then concluded by showing performance results and a comparison against existing link prediction packages.

\section{RELATED WORK}

Several researchers in the area of link prediction have released implementations of their methods (Clauset et al., 2008; Guimerà and Sales-Pardo, 2009; Liu et al., 2013; Papadopoulos et al., 2015; Muscoloni and Cannistraci, 2017). These implementations are, naturally but inconveniently, written in different languages and offer diverse modalities of interaction with the user, which complicates their effective use. There is also a limited number of packages that provide unified interfaces to implementations of topological ranking methods. The R package linkprediction (Bojanowski and Chrol, 2019), for instance, includes the implementation of the most important topological similarity algorithms. It offers a single method to compute the score of negative links using a specified similarity index. This package has several limitations, however. First, it only accepts connected undirected networks, which may be highly constraining as most real networks are disconnected. Since the package computes all negative links' scores, the size of networks that the package can handle is also limited. Furthermore, linkprediction does not offer any performance evaluation or test data generation functionalities. Other available packages include the commercial graph platform Neo4J (Neo4J, 2019), and NetworkX (Hagberg et al., 2019), which both contain the implementation of a limited number of topological ranking methods. The Python package linkpred (linkpred, 2020) contains the implementation of a number of topological similarity methods and also global methods, including rooted PageRank, Katz index (Katz, 1953), and SimRank (Jeh and Widom, 2002). The library does not, however, support parallel and distributed implementations, nor does it support performance evaluation functionalities.

GEM (Goyal and Ferrara, 2018b,a) is a Python package that implements many state-of-the-art graph embedding techniques, including Locally Linear Embedding (Roweis and Saul, 2000), Laplacian Eigenmaps (Belkin and Niyogi, 2001), Graph Factorization (Koren et al., 2009; Ahmed et al., 2013), Higher-Order Proximity preserved Embedding (HOPE) (Ou et al., 2016), Structural Deep Network Embedding (SDNE) (Wang et al., 2016), and node2vec (Grover and Leskovec, 2016). It also includes several similarity measures that can be used in combination with these embedding algorithms to predict links. GEM is, however, more focused on graph embedding techniques than link prediction and, as such, does not include other types of link prediction methods such as topological similarity and probabilistic methods. SNAP (Stanford Network Analysis Platform) (Leskovec and Sosič, 2016), which is a generalpurpose network analysis library, also includes an implementation of node2vec and GraphWave (Donnat et al., 2018). Like GEM, SNAP is not dedicated to link prediction, and apart from its graph embedding algorithms, it includes only a limited number of topological similarity measures as part of its experimental components (snap-exp). Another general-purpose network analysis library is the Python package scikitnetwork (Bonald et al., 2020), which contains the implementation of a number of local methods and graph embedding algorithms.

Given the importance of link prediction and the wide range of existing and potential applications, the currently available software packages clearly lack functionality and performance. Arguably, this stateof-affairs limits the successful application of existing algorithms to real-life problems and the rigorous testing of newly proposed methods. LinkPred aims at filling this gap that separates existing research from efficient software implementations. Table 1 contains a comparison in terms of functionality between LinkPred and the main open-source packages used for link prediction. The architecture of LinkPred and the functionalities shown in Table 1 are discussed in detail in the next section.

\section{ARCHITECTURE AND FUNCTIONALITIES}

LinkPred aims at filling the existing gap between research and efficient software implementations of link prediction algorithms. As shown in Figure 1, it offers functionalities at various levels to help use, 
Table 1. Comparison of LinkPred against the most important free/open-source link prediction software packages.

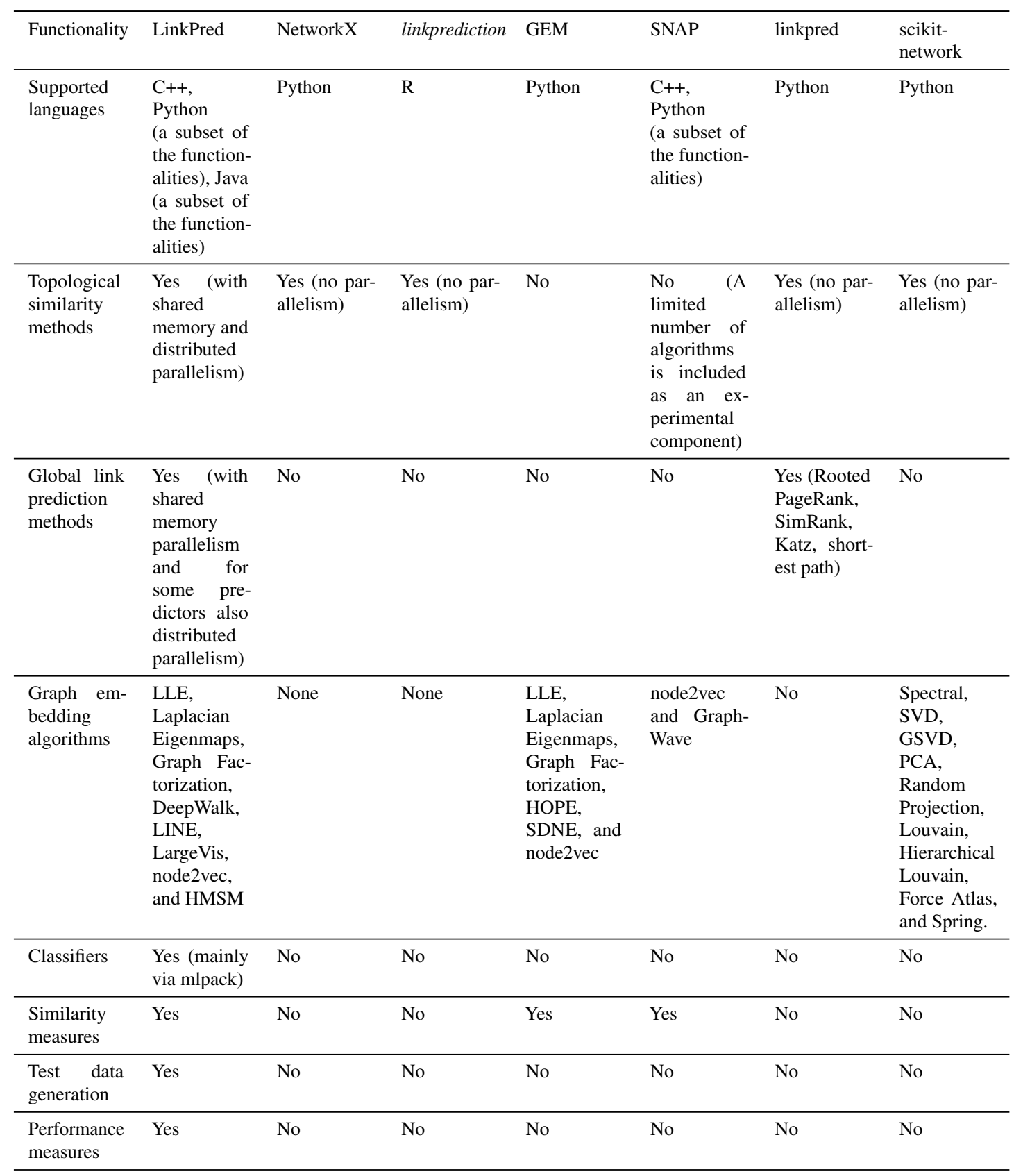

implement and test link prediction methods. In this section, a brief description of the functionalities available in LinkPred is given. More details can be found in the library user guide.

\section{Core components}

At the core of LinkPred lie efficient data structures for storing and accessing network data. These include the classes Unetwork and DNetwork used to represent undirected and directed networks, respectively. These structures allow efficient access to nodes, edges, and non-existing edges through C++-style iterators. Also included are auxiliary data structures such as full and sparse node and edge maps. 


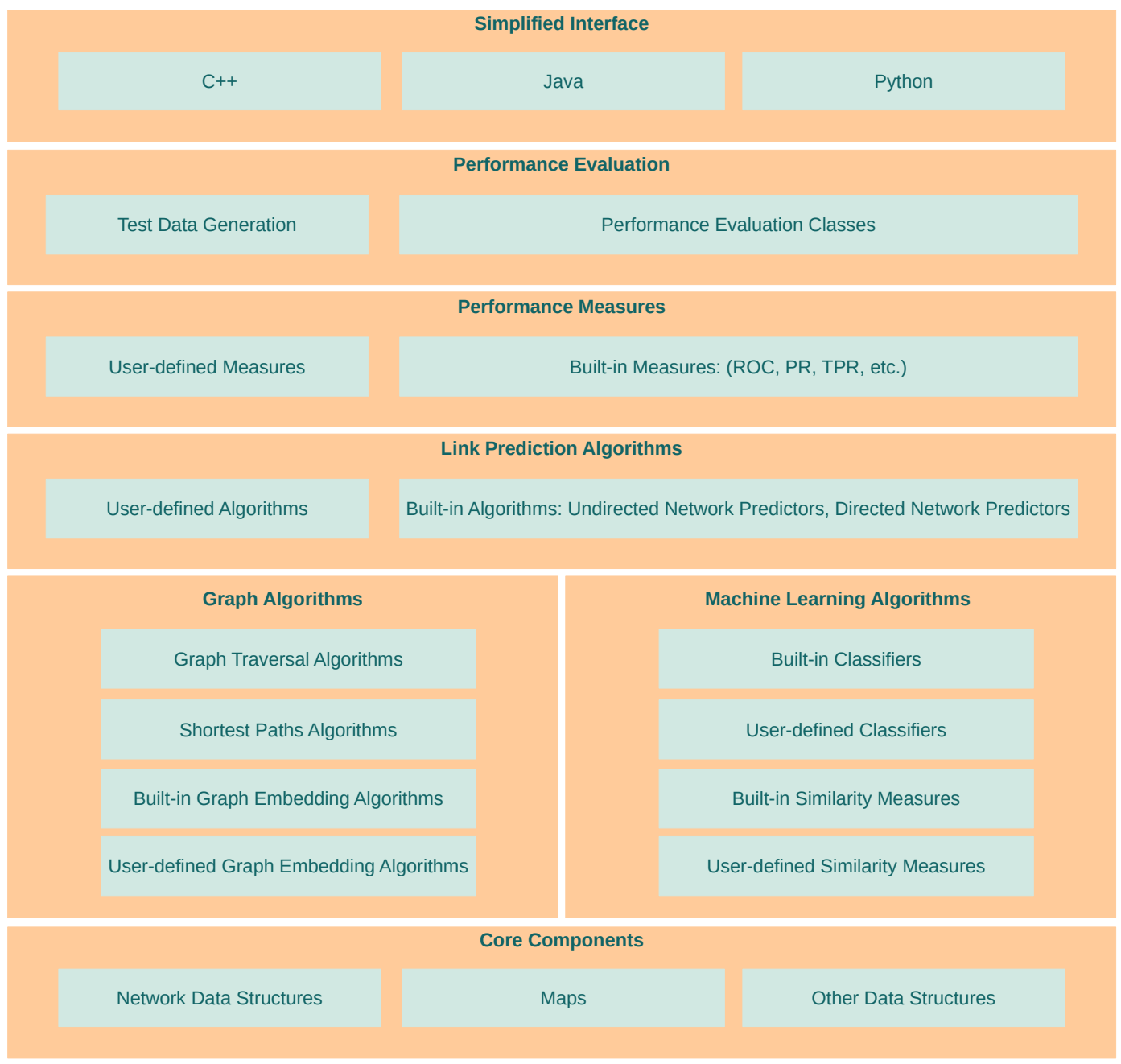

Figure 1. Architecture of LinkPred.

\section{The network data structures}

The life cycle of a network has two distinct phases. In the pre-assembly phase, it is possible to add nodes and edges to the network. It is also possible to access nodes and translate external labels to internal IDs and vice versa. However, most functionalities related to accessing edges are not yet available. As a result, the network at this stage is practically unusable. To be able to use the network, it is necessary to assemble it first. Once assembled, no new nodes or edges can be added (or removed) to the network. The network is then fully functional and can be passed as an argument to any method that requires so.

To build a network, an empty network is first created by calling the default constructor:

UNetwork<> net;

Most classes in LinkPred manipulate networks through smart pointers for efficient memory management. To create a shared pointer to a Unetwork object:

auto net $=$ std: : make_shared $<$ UNetwork $<>>()$;

Notice that the class unetwork is a class template, which is instantiated with the default template arguments. In this default setting, the labels are of type std: :string, whereas internal IDs are of type unsigned int, but unetwork can be instantiated with several other data types if wanted. For instance, the labels can be of type unsigned int, which may reduce storage size in some situations.

Adding nodes is achieved by calling the method addNode, which takes as parameter the node label and returns an std: :pair containing, respectively, the node ID and a Boolean which is set to true if the node is newly inserted, false if the node already exists. The nodes IDs are guaranteed to be contiguous in 
$0, \ldots, n-1$, where $n$ is the number of nodes.

auto res $=$ net. addNode (label);

auto id = res.first; // This the node ID

bool inserted = res.second; // Was the node inserted or did it already exist?

The method addEdge is used to create an edge between two nodes specified by their IDs (not their labels):

net.addEdge $(i, j)$;

subsection

The last step in building the network is to assemble it:

net. assemble();

The method assemble initializes the internal data structures and makes the network ready to be used.

Nodes can be accessed through iterators provided by nodesBegin () and nodesEnd (). For convenience, the iterator points to a pair, the first element of which is the internal ID, whereas the second is the external label.

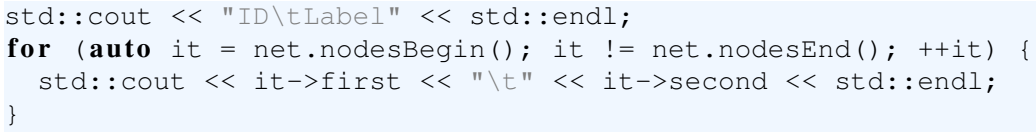

Alternatively, one can iterate over labels in a similar way using the iterators labelsBegin () and labelsEnd ():

It is also possible to translate labels to IDs and vice versa using getID (label) and getLabel (id) respectively. Oftentimes, one would want to iterate over a random sample of nodes instead of the whole set. This can be easily done using the two methods rndNodesBegin and rndNodesEnd.

Information on edges can only be accessed after assembling the network. One way to access edges is to iterate over all edges in the network. This can be done using the method edgesBegin () and edgesEnd (). As it is the case with nodes, it is possible to access a random sample of edges using rndEdgesBegin and rndEdgesEnd. LinkPred offers the possibility to iterate over negative links in the same way one iterates over positive edges. This can be done using the method nonEdgesBegin () and nonEdgesEnd():

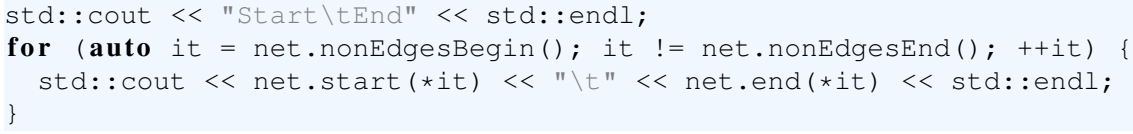

It is also possible to iterate over a randomly selected sample of negative links using rndNonEdgesBegin and rndNonEdgesEnd.

To represent directed networks, LinkPred offers the class DNetwork, which offers a very similar interface to UNetwork.

\section{Maps}

Maps are a useful way to associate data with nodes and edges. Two types of maps are available in LinkPred: node maps (class NodeMap) and edge maps (class EdgeMap), both member of unetwork. The first assigns data to the network nodes, whereas the latter maps data to edges (see Figure 2 for an example).

Creating a node map is achieved by calling the method createNodeMap on the network object. This is a template method with the mapped data type as the only template argument. For example, to create a node map with data type double over the network net:

auto nodeMap $=$ net . template createNodeMap $<$ double $>()$;

Creating an edge map can be done in a similar way:

auto edgeMap $=$ net. template createEdgeMap $<$ double $>()$; 


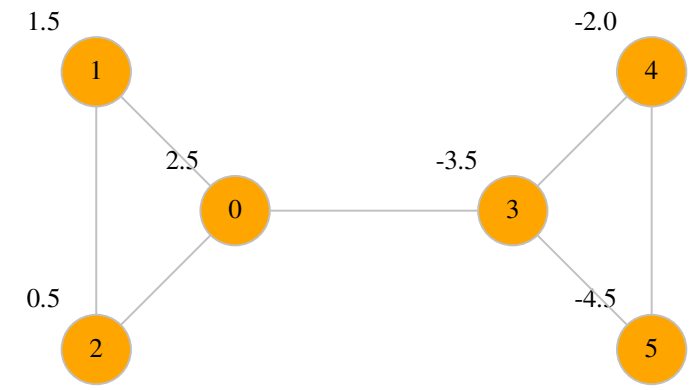

A

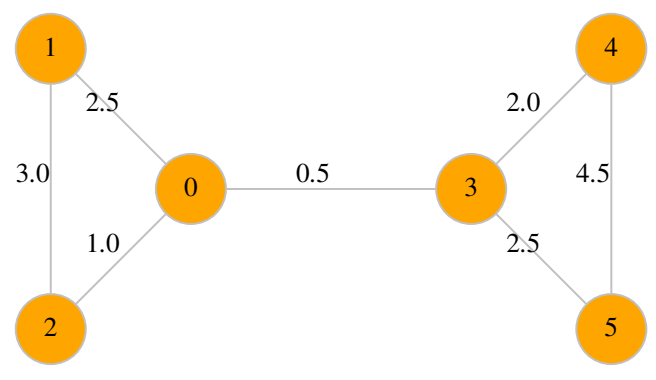

B

Figure 2. A node map associate values to nodes (A), whereas an edge map associates values to edges (B).

Both NodeMap and EdgeMap offer the same interface, which in fact is similar to std: :map. This includes the operator [ ], the methods at, begin, end, cbegin and cend. From the performance point of view, NodeMap offers constant time access to mapped values, whereas EdgeMap requires logarithmic time access $(O(\log m), m$ being the number of edges).

If a node map is sparse, that is, has non-default values only on a small subset of the elements, it is better to use a sparse node map. To create a sparse node map:

auto nodeSMap $=$ net. template createNodeSMap $<$ double $>(0.0)$;

Notice that the method takes as input one parameter that specifies the map's default value (in this case, it is 0.0 ). Hence, any node which is not explicitly assigned a value is assumed to have the default value 0.0 .

\section{Graph algorithms}

To facilitate the implementation of link prediction algorithms, LinkPred comes with a set of graphalgorithmic tools such as efficient implementations of graph traversal, shortest path algorithms, and graph embedding methods.

\section{Graph traversal and shortest paths algorithms}

LinkPred provides two classes for graph traversal: BFS, for Breadth-First traversal, and DFS for Depth-First traversal. They both inherit from the abstract class GraphTraversal, which declares one virtual method traverse. It takes as parameter the source node from where the traversal starts and a reference to a NodeProcessor object, which is in charge of processing nodes sequentially as they are visited.

In addition to graph traversal routines, LinkPred contains an implementation of Dijkstra's algorithm for solving the shortest path problem. To use it, it is first necessary to define a length (or weight) map that specifies the length associated with every edge in the graph. A length map is simply a map over the set of edges, that is, an object of type EdgeMap which can take integer or double values. The class Dijkstra offers two methods for computing distances:

- The method getShortestPath, which computes and returns the shortest path between two nodes and its length.

- The method getDist, which returns the distance between a source node and all other nodes. The returned value is a node map, where each node is mapped to a pair containing the distance from the source node and the number of edges in the corresponding shortest path.

Both methods run Dijkstra's algorithm, except that getShortestPath stops once the destination node is reached, whereas getDist continues until all reachable nodes are visited.

Computing shortest-path distances in large networks requires not only considerable time but also significant space resources. Consequently, efficient management of memory is necessary to render the task feasible in such situations. The abstract class NetDistCalculator provides an interface for an additional layer over the class Dijkstra which facilitates its use and can serve to manage memory usage. A NetDistCalculator object is associated with a single length map and provides two methods for computing distances: 
- getDist ( $i, j$ ): Computes and returns the distance between the two nodes $i$ and $j$. The returned value is an std: :pair, with the first element being the distance, whereas the second is the number of hops in the shortest path joining the two nodes.

- getDist (i): Computes and returns a node map containing the distances from node $i$ to all other nodes in the network.

LinkPred has two implementations of NetDistCalculator: ESPDistCalculator, an exact shortest path distance calculator which caches distances according to different strategies to balance memory usage and computation, and ASPDistCalculator, an approximate shortest path distance calculator. The approximation used in ASPDistCalculator works as follows. A set $\mathscr{L}$ of nodes called landmarks is selected, and the distance from each landmark to all other nodes is pre-computed and stored in memory. The distance between any two nodes $i, j$ is then approximated by:

$$
d_{i j} \simeq \min _{k \in \mathscr{L}}\left[d_{i k}+d_{k j}\right] .
$$

The landmarks are passed to ASPDistCalculator object using the method setLandmarks. Naturally, by increasing the number of landmarks, more precision can be obtained, be it though at a higher computational and memory cost.

\section{Graph embedding algorithms}

Graph embedding consists in transforming the graph's nodes and edges into elements of a low-dimensional vector space while preserving, as much as possible, its structural properties (Goyal and Ferrara, 2018c). It is a problem with important applications in various fields, including link prediction (Goyal and Ferrara, 2018c; Kazemi and Poole, 2018; Alharbi et al., 2016; Wang et al., 2016), product recommendation (Koren et al., 2009), data visualization (van der Maaten and Hinton, 2008; Tang et al., 2016b; Cao et al., 2016), and node classification (Bhagat et al., 2011; Tang et al., 2016a).

LinkPred contains several state-of-the-art graph embedding algorithms, some of which are implemented from scratch, whereas others are based on publicly available implementations. These include methods based on matrix decomposition, namely Locally Linear Embedding (Roweis and Saul, 2000) implemented in the class LIE, Laplacian Eigenmaps (Belkin and Niyogi, 2001) implemented in the class LEM, and Matrix Factorization (Koren et al., 2009) (also referred to as Graph Factorization in (Goyal and Ferrara, 2018c; Ahmed et al., 2013)) implemented in the class MatFact. Also available are methods based on random walks, including DeepWalk (Perozzi et al., 2014) implemented in the class DeepWalk, Large Information Networks Embedding (LINE) (Tang et al., 2015), implemented in the class LINE, LargeVis (Tang et al., 2016b) implemented in the class LargeVis, and node2vec (Grover and Leskovec, 2016), which is implemented in the class Node2Vec. Additionally, the librray includes the implementation of the Hidden the Metric Space Model (HMSM) embedding method (Alharbi et al., 2016) available through the class HMSM.

To provide a uniform interface, all embedding algorithms implemented in LinkPred inherit from the abstract class Encoder, which declares the following methods:

- The method init, which is first called to initialize the internal data structures of the encoder. This is a pure virtual method of the class Encoder and must be implemented by derived classes.

- Once the encoder is initialized, the method encode, also a pure virtual method, is called to perform the embedding. This step typically involves solving an optimization problem, which can be computationally intensive both in terms of memory and CPU usage, especially for very large networks. The dimension of the embedding space can be queried and set using getDim and setDim respectively.

- The node embedding or the node code, which is the vector of coordinates assigned to the node, can be obtained by calling the method getNodecode. The edge code is by default the concatenation of its two nodes' codes and can be obtained using getEdgecode. Hence, in the default case, the edge code dimension is double that of a node. Classes that implement the Encoder interface may change this default behavior if desired. The user can query the dimension of the edge code using the method getEdgeCodeDim. 


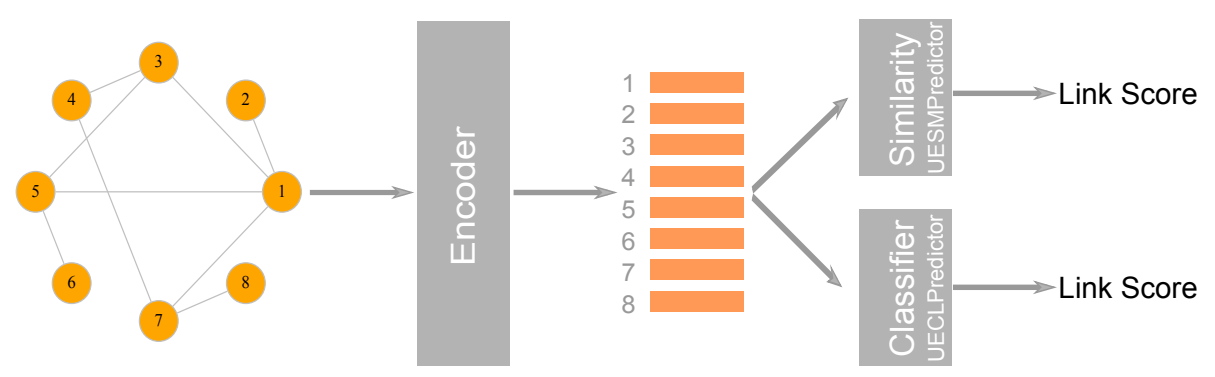

Figure 3. The first stage in a graph embedding method is accomplished by an encoder class which uses a graph embedding algorithm to . In the class UESMPredictor, this is followed by a similarity measure to predict link scores, whereas UESMPredictor uses a classifier to make the prediction.

Having a unified interface for encoders allows embedding algorithms to be easily combined with different classifiers and similarity measures to obtain various link prediction methods, as explained in the next sections. It also allows users to use their own embedding algorithms to build and test new link prediction methods.

\section{Machine learning algorithms}

The library contains the implementations of several classifiers and similarity measures that can be combined with graph embedding algorithms (see the previous section) to build a variety of link prediction methods. Available classifiers, most of which are derived from mlpack (Curtin et al., 2013), include logistic regression, feed-forward neural networks, linear support vector machine, and Naive Bayes classifier. All binary classifiers in LinkPred implement the interface Classifier, which provides two important methods: the method learn which trains the classifier on a training set, and the method predict which predicts the output for a given input.

Similar to classifiers, all similarity measures in LinkPred inherit from the abstract class simMeasure, which defines one method, sim, which computes the similarity between two input vectors. Implemented similarity measures include cosine similarity, dot product similarity, $L_{1}, L_{2}$ and $L_{p}$ similarity, and Pearson similarity.

\section{Link predictors}

LinkPred includes a large selection of link prediction algorithms which can be broadly classified into three categories: topological similarity methods, global methods, and graph-embedding techniques. In terms of topological similarity predictors, the library contains the implementations of the most known algorithms existing in the literature, including Common Neighbors, Adamic-Adard, Resource Allocation, Cannistraci Resource Allocation, and Jackard Index, among other predictors. (Liben-Nowell and Kleinberg, 2007; Newman, 2001; Jaccard, 1901; Adamic and Adar, 2003; Ravasz et al., 2002; Papadimitriou et al., 2012; Liu and Lü, 2010; Lichtenwalter et al., 2010; Yang et al., 2015b,c; Zhu and Xia, 2015; Muscoloni and Cannistraci, 2017; Cannistraci et al., 2013; Daminelli et al., 2015). Due to their local nature, these algorithms can scale to very large networks, especially when executed on distributed architectures. Addiitonally, the library includes several state-of-the-art global link predictors, such as SBM (Guimerà and Sales-Pardo, 2009), HRG (Clauset et al., 2008), FBM (Liu et al., 2013), HyperMap (Papadopoulos et al., 2012, 2015) and the popularity-similarity method proposed in (Kerrache et al., 2020).

LinkPred also supports link prediction algorithms based on graph embedding, where the network is first embedded into a low dimensional vector space, whereby nodes are assigned coordinates in that space while preserving the network's structural properties. These coordinates can be used either to compute the similarity between nodes or as features to train a classifier to discriminate between existing edges (the positive class) and non-existing edges (the negative class) (Goyal and Ferrara, 2018c). LinkPred provides two classes that can be used to build link prediction algorithms based on graph embedding: the class UECLPredictor, which combines an encoder (a graph embedding algorithm) and a classifier, and the class UESMPredictor, which pairs the encoder with a similarity measure as illustrated in Figure 3.

In addition to algorithms for undirected networks, several adaptations of topological similarity methods to directed networks are available as well. The library offers a unified interface for all link prediction algorithms, simplifying the use and comparison of different prediction methods. The interface is called 
ULPredictor for predictors in undirected networks and DLPredictor for those in directed networks. Most implemented predictors support shared-memory parallelism, and a large number of them support distributed memory parallelism, allowing LinkPred to take advantage of the power of HPC clusters to handle very large networks.

\section{The predictor interface}

As stated above, all link predictors for undirected networks must inherit from the abstract class uLPredictor . It declares three important pure virtual methods that the derivative classes must implement:

- The method void init(): This method is used to initialize the predictor's state, including any internal data structures.

- The method void learn (): In algorithms that require learning, it is in this method that the model is built. The learning is separated from prediction because, typically, the model is independent of the set of edges to be predicted.

- The method double score (Edge const $\& e$ ): returns the score of the edge e (usually a non-existing edge).

In addition to these three basic methods, ULPredictor declares the following three virtual methods, which by default use the method score to assign scores to edges, but which can be redefined by derived classes to achieve better performance:

- The method void predict (EdgeRndIt begin, EdgeRndIt end, ScoreRndIt scores): In this method, the edges to be predicted are passed to the predictor in the form of a range (begin, end) in addition to a third parameter (scores) to which the scores are written. This is a virtual method that uses the method score to assign scores to edges and can be redefined by derived classes to provide better performance.

- The method std: :pair<NonEdgeIt, NonEdgeIt> predictNeg(ScoreRndIt scores) predicts the score for all negative (non-existing) links in the network. The scores are written into the random output iterator scores. The method returns a pair of iterators begin and end to the range of non-existing links predicted by the method.

- The methodstd: :size_t top(std:size_t k, EdgeRndoutIt eit, ScoreRndIt sit) finds the $k$ negative edges with the top scores. The edges are written to the output iterator eit, whereas the scores are written to sit.

The class ULPredictor offers default implementations for the methods top, predict and predictNeg. Sub-classes may use these implementations or redefine them to achieve better performance.

The abstract class DLPredictor plays the same role as ULPredictor but for link predictors in directed networks. It offers the same interface as the latter but with different default template arguments and methods implementation.

\section{Performance evaluation}

LinkPred offers a set of tools that help to streamline the performance evaluation procedure. This includes data setup functionalities, which can be used to create test data by removing and adding edges to ground truth networks. The library also includes efficient implementations of the most important performance measures used in link prediction literature, including the area under the receiver operating characteristic (ROC) curve, the area under the precision-recall (PR) curve, and top precision. The area under the PR curve can be computed using two integration methods: the trapezoidal rule, which uses a linear interpolation between the PR points, and the more accurate nonlinear interpolation method proposed in (Davis and Goadrich, 2006). In addition to performance measures implementations, LinkPred contains helper classes, namely PerfEvaluator and Perfevalexp, that facilitate the comparative evaluation of multiple link prediction algorithms using multiple performance measures.

All performance measures inherit from the abstract class Perfmeasure. The most important method in this class is eval which evaluates the value of the performance measure. The performance measure results are written to an object of type PerfResults passed as a parameter of the method. The class 


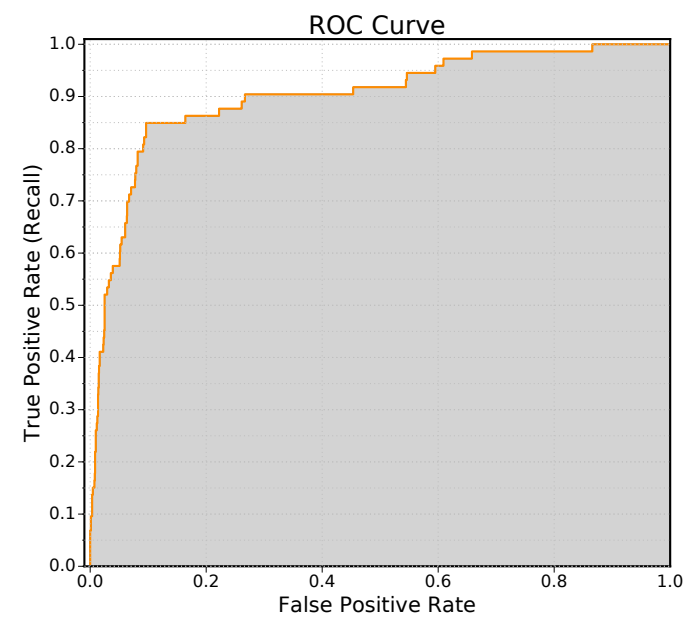

A

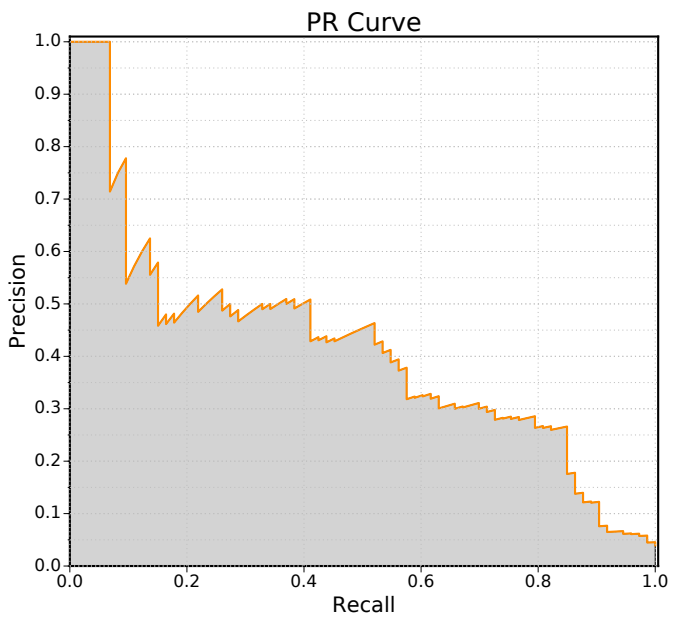

B

Figure 4. Example of performance curves generated by LinkPred (the plots are created using an external tool). The area under the curve (shown in gray) is the value associated with the performance curve.

PerfResults is defined as std: :map<std: :string, double>, which allows the possibility of associating several result values with a single performance measure.

An important class of performance measures is performance curves such as ROC and PR curves. They are represented by the abstract class PerfCurve, which inherits from the class Perfmeasure. The class PerfCurve defines a new virtual method getcurve, which returns the performance curve in the form of an std: : vector of points. In the remainder of this section, more details of the performance measures implemented in LinkPred are presented.

\section{Receiver operating characteristic curve (ROC)}

One of the most important performance measure used in the field of link prediction is the receiver operating (ROC) curve, in which the true positive rate (recall) is plotted against the false positive rate. The ROC curve can be computed using the class Roc. Figure 4 (A) shows an example ROC curve obtained using this class.

The default behavior of the Roc performance measure is to compute the positive and negative edge scores and then compute the area under the curve, which may lead to memory issues with large networks. To compute the area under the curve without storing both types of scores, the class ROC offers a method that streams scores without storing them. To enable this method, call setSt rmEnabled (bool) on the ROC object. To specify which scores to stream use the method setstrmneg (bool). By default, the negative scores are streamed, while the positive scores are stored. Passing false to setStrmNeg switches this. In addition to consuming little memory, the streaming method supports distributed processing (in addition to shared memory parallelism), making it suitable for large networks.

\section{Precision-recall curve}

The precision-recall (PR) curve is also a widely used measure of link prediction algorithms' performance. In this curve, the precision is plotted as a function of the recall. The PR curve can be computed using the class PR. The area under the PR curve can be computed using two integration methods:

- The trapezoidal rule which assumes a linear interpolation between the PR points.

- Nonlinear interpolation as proposed by Jesse Davis and Mark Goadrich (Davis and Goadrich, 2006).

The second method is more accurate, as linear integration tends to overestimate the area under the curve (Davis and Goadrich, 2006). Furthermore, the implementation of Davis-Goadrich nonlinear interpolation in LinkPred ensures little to no additional cost compared to the trapezoidal method. Figure 4 (B) shows an example PR curve obtained using this class PR. 


\section{General performance curves}

LinkPred offers the possibility of calculating general performance curves using the class GCurve. A performance curve is, in general, defined by giving the $x$ and $y$ coordinates functions. These are passed as parameters, in the form of lambdas, to the constructor of the class GCurve. The associated performance value is the area under the curve computed using the trapezoidal rule (linear interpolation). For example, the ROC curve can be defined as:

GCurve<> cur(fpr, rec, "ROC");

The two first parameters of the constructors are lambdas having the signature:

double (std: :size_t tp, std:size_t fn, std:size_t tn, std:size_t fp, std:size_t P, std: :size_t $N$ )

\section{Top precision}

The top precision measure is defined as the ratio of true positives within the top $l$ scored edges, $l>0$ being a parameter of the measure (usually $l$ is set to the number of links removed from the network). Top precision is implemented by the class TPR, and since it is not a curve measure, this class inherits directly from PerfMeasure. The class TPR offers two approaches for computing top-precision. The first approach requires computing the score of all negative links, whereas the second approach calls the method top of the predictor. The first approach is, in general, more precise but may require more memory and time. Consequently, the second approach is the performance measure of choice for very large networks.

\section{Simplified interface and bindings}

The simplified interface provides the essential functionalities available in LinkPred via a small number of easy-to-use classes. These classes are very intuitive and can be used with a minimum learning effort. They are ideal for initial use of the library and exploring its main functionalities. Java and Python bindings for the simplified interface are also available, facilitating the library's use by users who are more comfortable using these languages. The simplified interface contains two main classes: Predictor, which allows computing the scores for an input network using all available link prediction algorithms, and the class Evaluator, which can be used for performance evaluation. Also included are simple structures to store prediction and performance results. These classes are designed in a simple way that allows uniform usage across different programming languages.

\section{EXAMPLE USE CASES}

This section describes four main use scenarios of the library. The first use case demonstrates the working of the simplified interface in different languages, which is typical for first-time use of the library or for users who prefer to use the library in Python or Java. The second scenario consists in computing the scores of all non-existing links in a network, which is the typical use case for a practitioner working on networked data. Researchers in link prediction are typically interested in implementing new link prediction algorithms, which is presented as the third use case, and evaluating their performance, which is use case number four.

\section{Using the simplified interface}

The first example program shows how to use the simplified interface to obtain the top $k$ ranked edges using Adamic Adar index in c++:

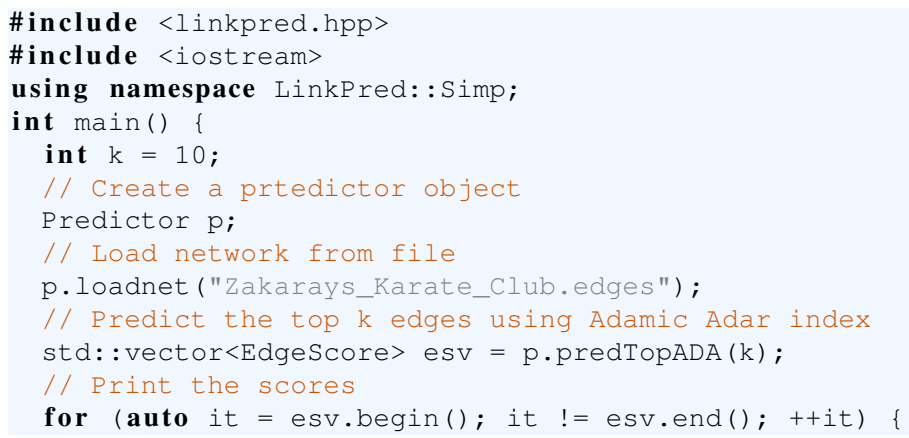




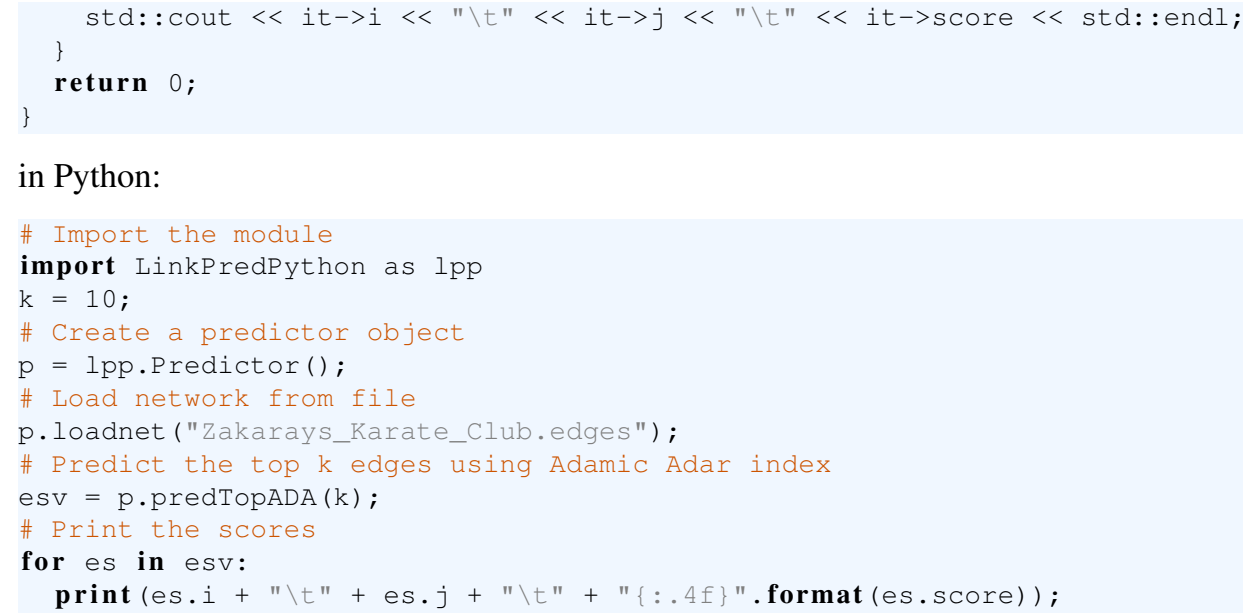

The output of these three programs is as follows:

$\begin{array}{lll}1 & 33 & 1.61374 \\ 1 & 34 & 2.71102 \\ 2 & 34 & 2.25292 \\ 3 & 32 & 1.67334 \\ 3 & 34 & 4.71938 \\ 5 & 6 & 1.99226 \\ 7 & 11 & 1.99226 \\ 8 & 14 & 1.8082 \\ 32 & 24 & 1.66562 \\ 24 & 25 & 1.63159\end{array}$

In the second example, the performance measure of several link prediction algorithms is evaluated by removing $10 \%$ of the links from the network and using it as a test set. The performance is assessed using two performance measures, area under the ROC curve, and top-precision. The code for this example in $\mathrm{C}++$ :

\#include <linkpred.hpp>

\#include <iostream>

using namespace LinkPred: Simp;

int main() \{

int nbRuns $=10$;

double edgeRemRatio $=0.1$;

// Create an evaluator object

Evaluator eval;

// Add predictors to be evaluated

eval.addCNE ( ) ; 


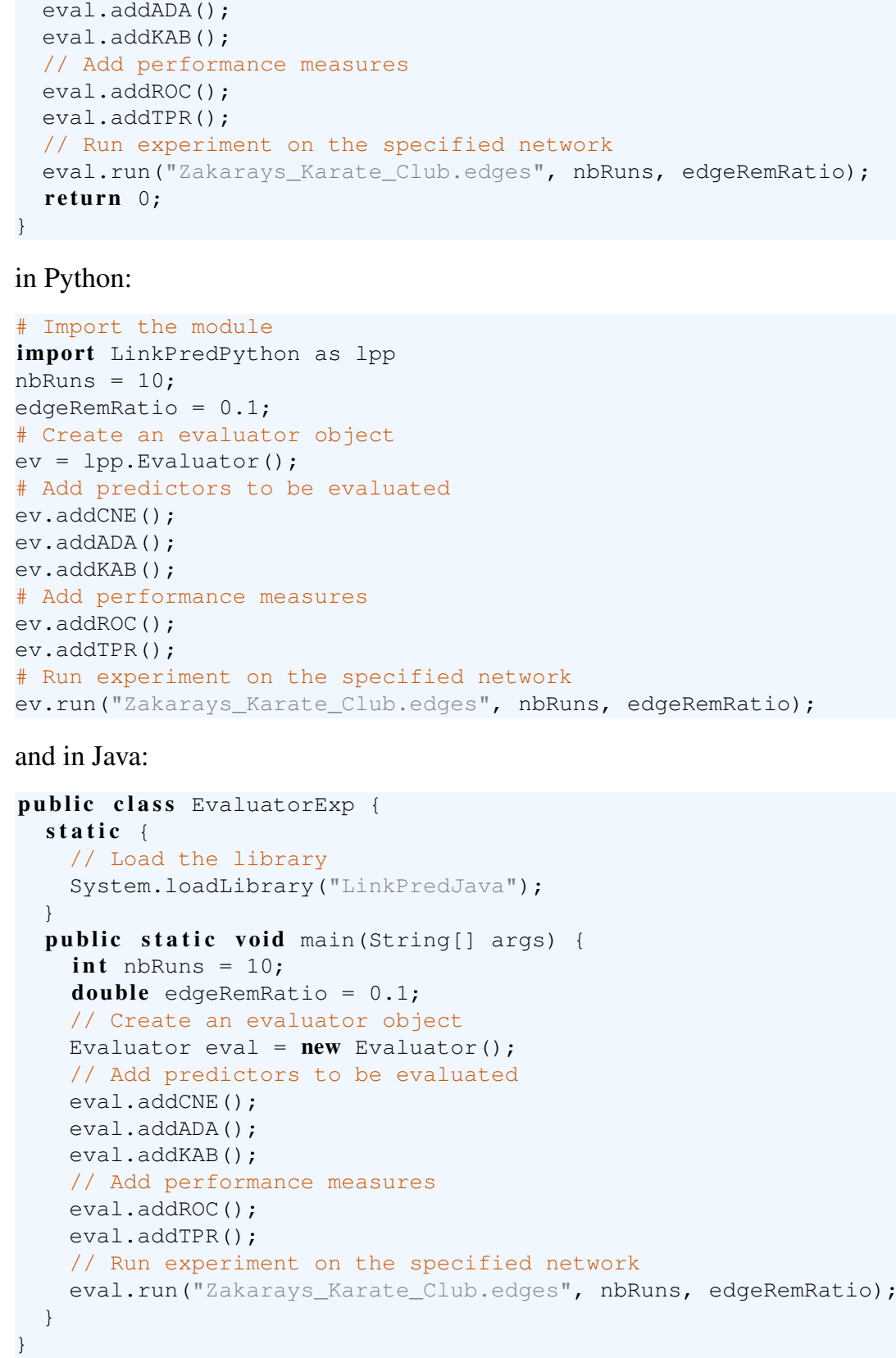

\section{Predicting missing links}

When dealing with networked data, a data scientist may be interested in reconstructing a network from partial observations or predicting future interactions. LinkPred offers two ways to solve such problems, 
computing the scores of all non-existing links and computing top $k$ edges, which may be more efficient for large networks. This section demonstrates how to perform both tasks.

The following code excerpt shows how to compute and print the scores of all non-existing links in a network using SBM. The observed network is passed as an argument to the constructor of the algorithm, which is then initialized by calling the method init. The learning process, if any, is triggered by a call to the method learn. The simplest way to obtain the score of a non-existing link is to call the method score, though other methods of the predictor interface may result in better performance.

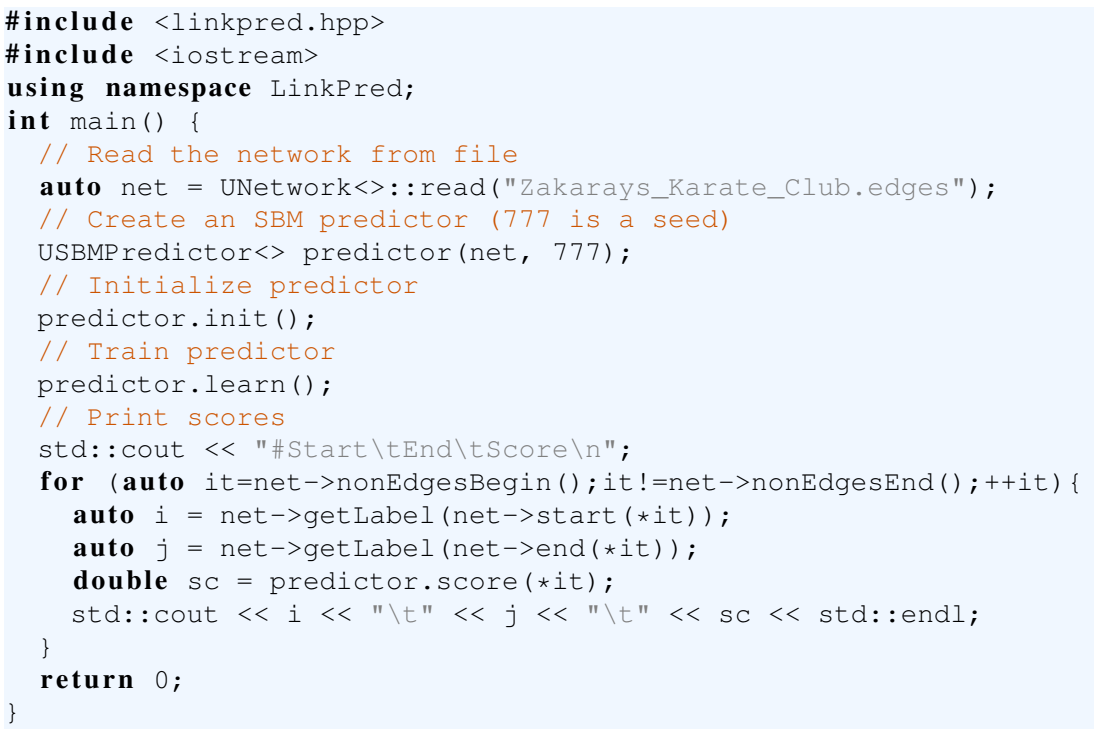

The first few lines of the output of this program are as follows:

$\begin{array}{lll}\text { \#Start } & \text { End } & \text { Score } \\ 1 & 31 & 0.208413 \\ 1 & 10 & 0.248398 \\ 1 & 28 & 0.229615 \\ 1 & 29 & 0.246439 \\ 1 & 33 & 0.316544 \\ 1 & 17 & 0.685567 \\ 1 & 34 & 0.315658 \\ 1 & 15 & 0.17834 \\ 1 & 16 & 0.178189 \\ \ldots & & \end{array}$

We can also use an embedding-classifier predictor. In the following code, the graph is embedded using node2vec and logistic regression is used to predict scores.

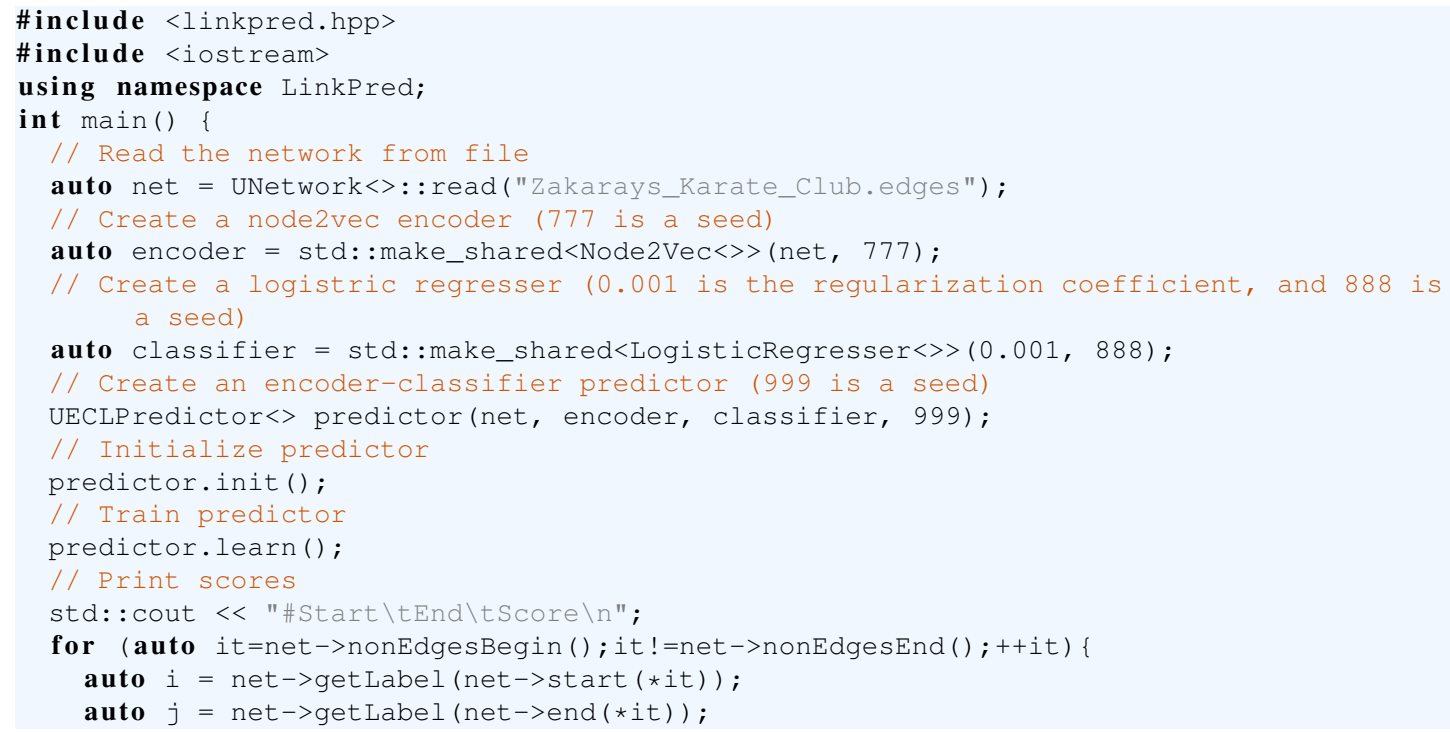




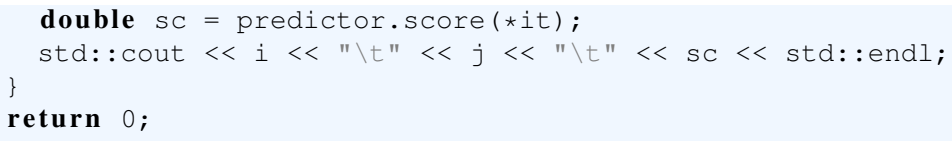

Instead of computing the scores of all non-existing links, it is possible to extract the top $k$ ranked edges only. Besides convenience, this approach may be the only viable option for very large networks due to memory considerations. Furthermore, for many prediction algorithms, particularly topological similarity methods, finding the top $k$ edges is much faster than computing the scores of all non-existing links. The following code shows how to find the top $k$ edges using Resource Allocation index.

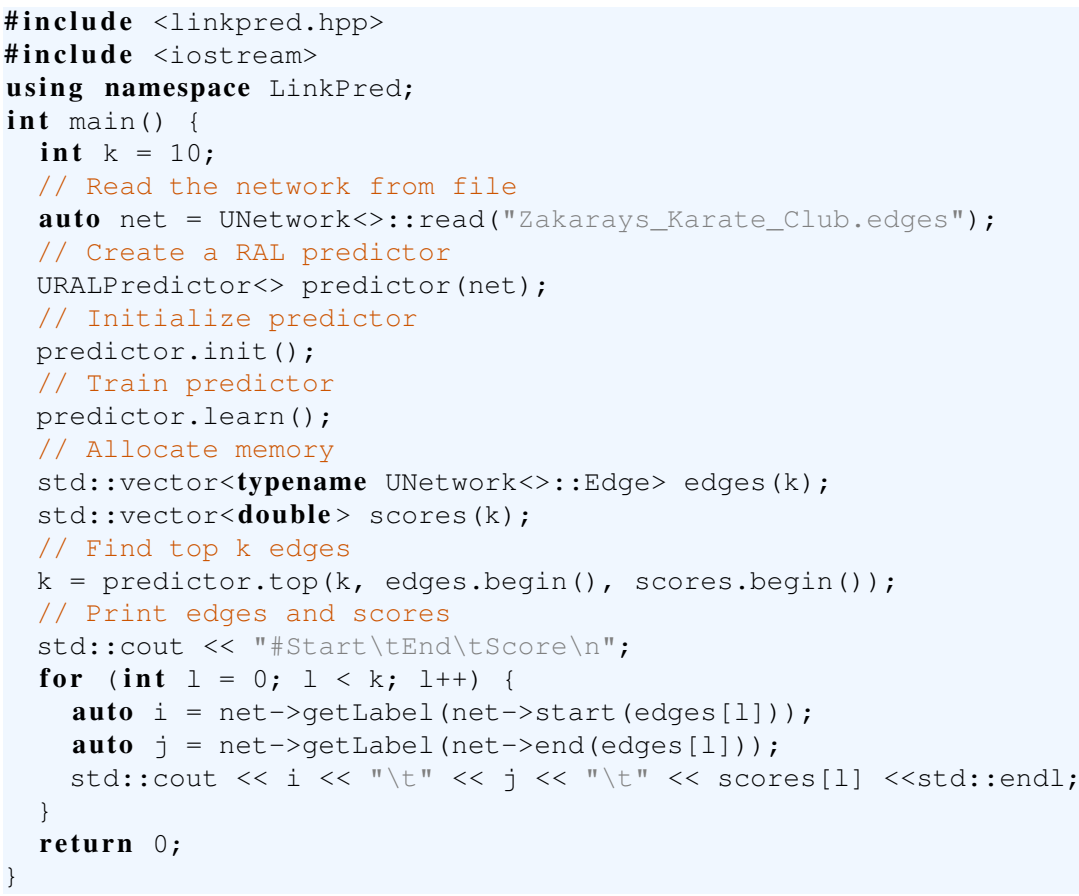

This is the output of this program:

$\begin{array}{lll}\text { Start } & \text { End } & \text { Score } \\ & 17 & 0.5 \\ & 34 & 0.9 \\ 2 & 34 & 0.783333 \\ 3 & 32 & 0.479167 \\ 3 & 34 & 1.56667 \\ 5 & 6 & 0.645833 \\ 3 & 11 & 0.645833 \\ 32 & 24 & 0.47549 \\ 8 & 26 & 0.533333 \\ 4 & 25 & 0.583333\end{array}$




\section{Implementing a new link prediction algorithm}

The first step in implementing a new link prediction algorithm is to inherit from ULPredictor and implement the necessary methods. For a minimal implementation, the three methods init, learn and score must at least be defined. To achieve better performance one may want to redefine the three other methods (top, predict and predictNeg).

Suppose one wants to create a very simple link prediction algorithm that assigns as score to $(i, j)$ the score $\kappa_{i}+\kappa_{j}$, the sum of the degrees of the two nodes. In a file named sdpredictor.hpp, write the following code:

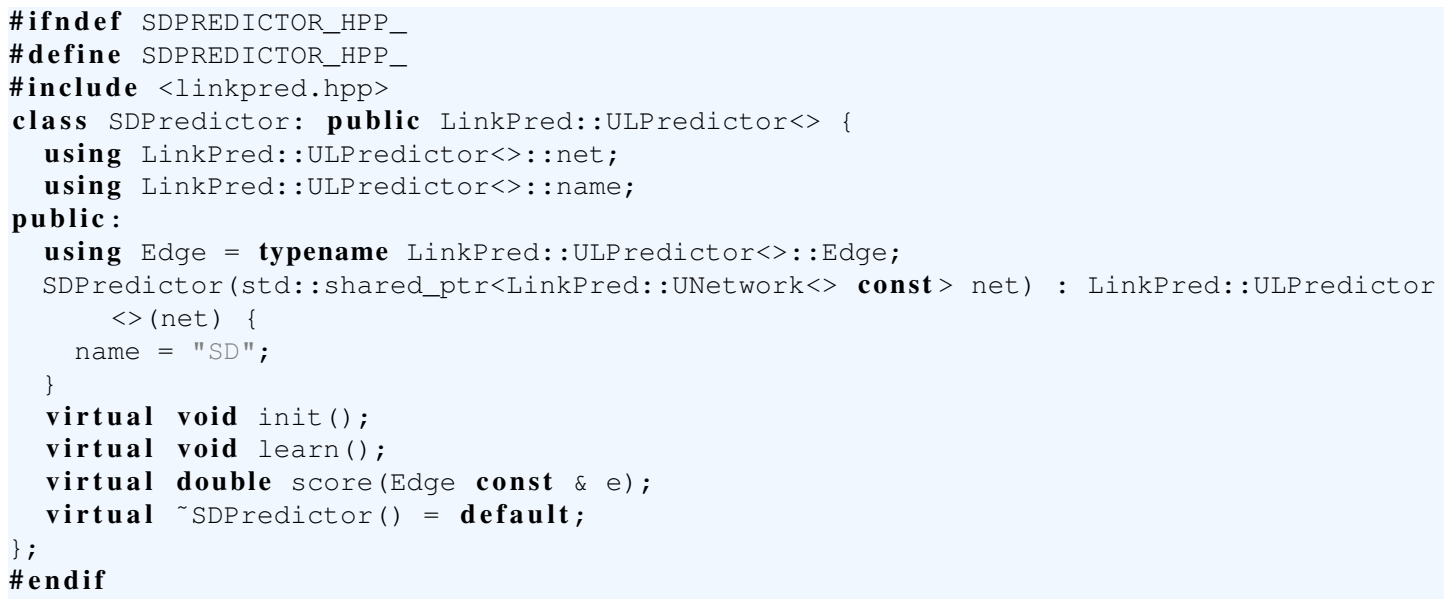

In a file named sdpredictor.cpp write the implementation of the inherited methods (note that this predictor does not require initialization or learning):

\#include "sdpredictor.hpp"

// No init required

void SDPredictor: :init() \{\}

// No training required

void SDPredictor: : learn() \{\}

// Here, we compute the score

double SDPredictor: :score(Edge const \& e) \{

auto $i=$ net->start $(e)$;

auto $j=$ net->end $(e)$;

$/ /$ Return the sum of degrees

This predictor is now ready to be used with LinkPred classes and methods including performance evaluating routines. For instance, it is possible to write a code that extracts the edges with the top scores as follows:

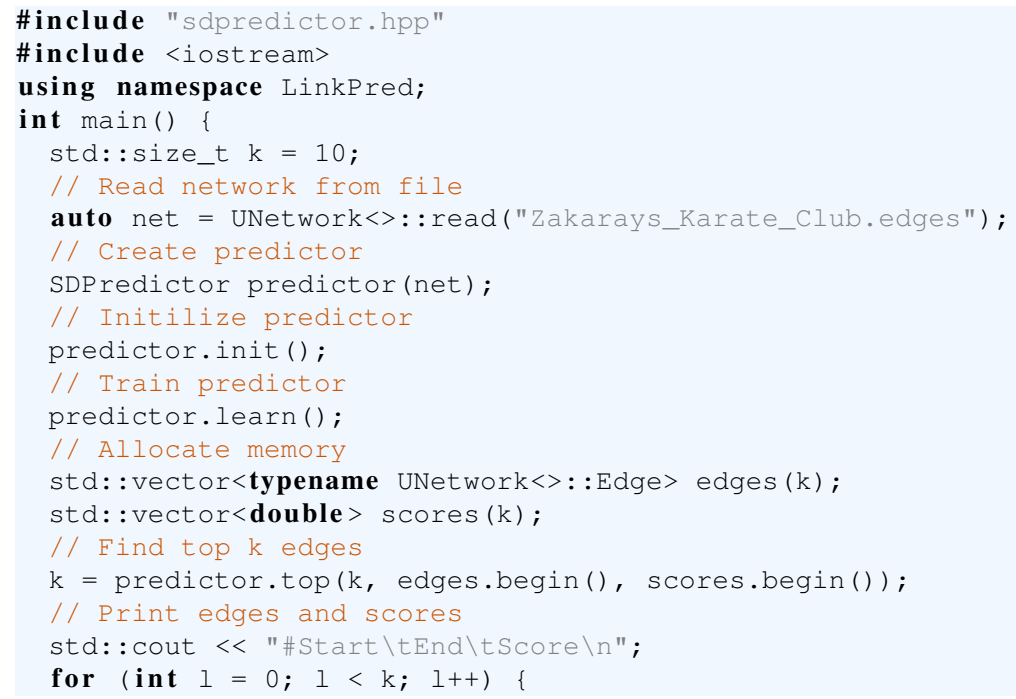




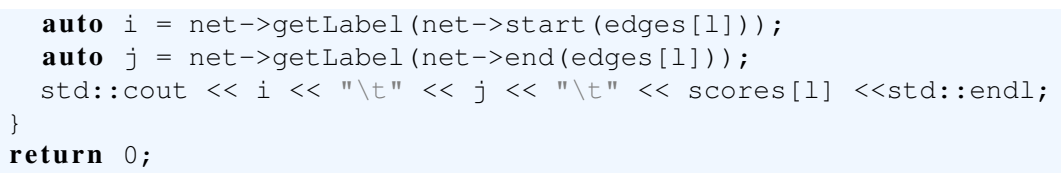

Upon compiling and executing this code, the output will be as follows (for compilation instructions, the reader is invited to consult the library user guide):

$\begin{array}{lll}\text { \# Start } & \text { End } & \text { Score } \\ 1 & 33 & 28 \\ 1 & 34 & 33 \\ 1 & 24 & 21 \\ 2 & 33 & 21 \\ 2 & 34 & 26 \\ 3 & 34 & 27 \\ 4 & 34 & 23 \\ 6 & 34 & 21 \\ 7 & 34 & 21 \\ 8 & 34 & 21\end{array}$

New link prediction algorithms can also be easily integrated into the library source code, as explained in detail in the library user guide.

\section{Performance evaluation}

Another use case scenario is evaluating and comparing the performance of link prediction algorithms. LinkPred offers several ways to achieve this, offering various degrees of control on the evaluation process. One such method is shown in the code sample below. Here, the user defines a factory class used to instantiate the prediction algorithms and performance measures. The parameters of the experiment, including the ratio of removed edges and the number of test runs, are passed through an object of type PerfeEvalexpDescp. The evaluation is finally conducted by passing the factory and parameter objects to an object of type PerfEvalExp then calling the method run.

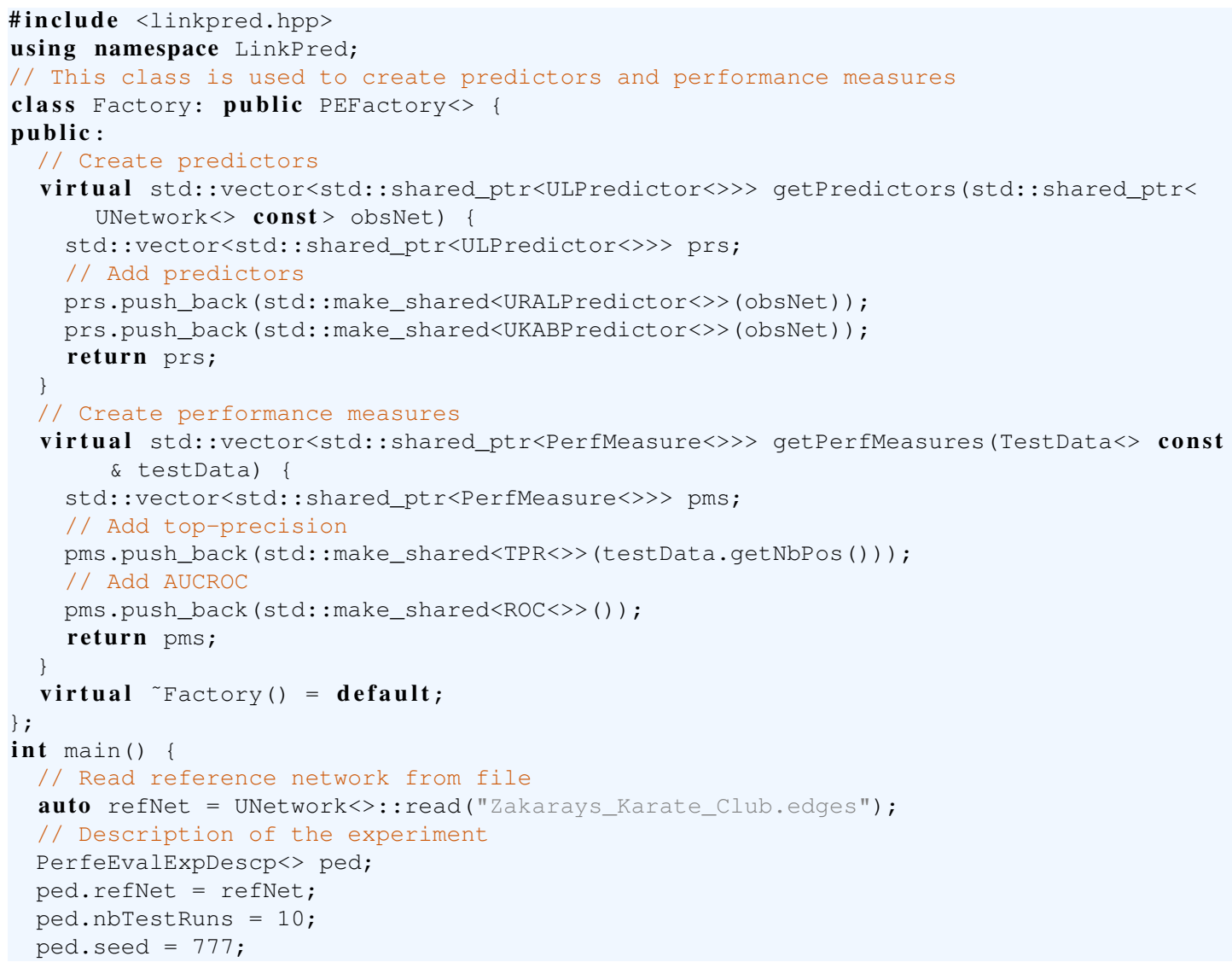




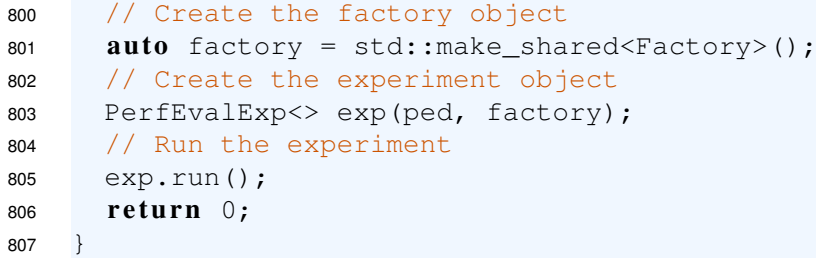

The output results for the first few iterations is a s follows:

$\begin{array}{lllll}\text { \#ratio } & \text { ROCKAB } & \text { ROCRAL } & \text { TPRKAB } & \text { TPRRAL } \\ 0.10 & 0.8615 & 0.8028 & 0.1250 & 0.1250 \\ 0.10 & 0.7943 & 0.7823 & 0.1250 & 0.1667 \\ 0.10 & 0.6945 & 0.6712 & 0.0000 & 0.0000 \\ 0.10 & 0.6417 & 0.6219 & 0.2500 & 0.1250 \\ 0.10 & 0.5817 & 0.5487 & 0.0000 & 0.0000 \\ 0.10 & 0.8527 & 0.8386 & 0.3750 & 0.3438 \\ 0.10 & 0.5705 & 0.5167 & 0.0000 & 0.0000 \\ 0.10 & 0.8834 & 0.8359 & 0.1250 & 0.1250 \\ 0.10 & 0.8962 & 0.8617 & 0.2500 & 0.1250 \\ 0.10 & 0.7650 & 0.7433 & 0.2500 & 0.2500\end{array}$

More use case examples can be found in the library documentation. These include using other link prediction algorithms, computing the scores of a specific set of edges, and other methods for computing the performance of one or several link prediction algorithms.

\section{EXPERIMENTAL RESULTS}

In addition to providing an easy interface to use, create and evaluate link prediction algorithms, LinkPred is designed to handle very large networks, which is a quality that is essential for most practical applications. To demonstrate the performance of LinkPred, its time performance is compared to that of the R package linkprediction and the Python packages linkpred, NetworkX and scikit-network. To conduct a fair and meaningful comparison, two issues are to be resolved. First, these packages do not implement the same set of algorithms, and only a limited number of topological similarity methods are implemented by all five libraries. Accordingly, the Resource Allocation index is chosen as the comparison task, since it is implemented by all five packages and exhibits the same network data access patterns as most local methods. The second issue that needs to be addressed is that the libraries under consideration offer programming interfaces with different semantics. For instance, scikit-network computes the score for edges given as input, whereas the R package linkprediction and Python packages Linkpred and NetworkX do not require input and instead return the scores of non-existing links. Furthermore, the Python package linkpred returns the scores of only candidate edges that have a non-zero score. To level the field, the comparison shall consist in computing the scores of all non-existing links, even those with zero scores. All networks used in this experiment are connected due to the restriction imposed by the package linkprediction. A description of these networks is given in Table 4 of the appendix. For the sake of fairness, parallelism is disabled in LinkPred, and all experiments are conducted on a single core of an Intel Core i7-4940MX CPU with $32 \mathrm{~GB}$ of memory. The time reported in Table 2 is the average execution time over ten runs, excluding the time required to read the network from file. The time for LinkPred is reported for C++ code and the Java and Python bindings. The results show that LinkPred is typically one to two orders of magnitudes faster than the other packages. This, of course, can in part be explained by the interpreted nature of Python and $\mathrm{R}$, but it also highlights the fact that link prediction is a computationally intensive task that is best handled by high-performance software that uses efficient data structures and algorithms. As shown in the table, the Java binding of LinkPred introduces a small overhead compared to its Python binding due to more complex data marshaling in the latter. Nevertheless, the Python binding is significantly faster than the Python packages and, except for a couple of networks, is also faster than linkprediction.

Table 3 shows the time taken by LinkPred to complete different link prediction tasks on various hardware architectures. It shows that the library can handle very large networks in relatively small amounts of time, even when the available computational resources are limited. 
Table 2. Time (in seconds) required to compute the score of all non-existing links using Resource Allocation index on a single core.

\begin{tabular}{llllllll}
\hline Network & $\begin{array}{l}\text { LinkPred } \\
(\mathrm{C}++)\end{array}$ & $\begin{array}{l}\text { LinkPred } \\
\text { (Java) }\end{array}$ & $\begin{array}{l}\text { LinkPred } \\
\text { (Python) }\end{array}$ & $\begin{array}{l}\text { Python } \\
\text { package } \\
\text { NetworkX }\end{array}$ & $\begin{array}{l}\text { R package } \\
\text { linkpredic- } \\
\text { tion }\end{array}$ & $\begin{array}{l}\text { Python pack- } \\
\text { age linkpred }\end{array}$ & $\begin{array}{l}\text { Python } \\
\text { package } \\
\text { scikit- } \\
\text { network }\end{array}$ \\
\hline $\begin{array}{l}\text { Political } \\
\text { Blogs }\end{array}$ & 0.02 & 0.03 & 0.14 & 1.83 & 3.70 & 0.68 & 3.13 \\
\hline Diseasome & 0.04 & 0.16 & 0.86 & 6.33 & 2.53 & 1.26 & 14.98 \\
\hline Email & 0.05 & 0.12 & 0.56 & 7.78 & 6.88 & 1.60 & 9.63 \\
\hline Web Edu & 0.14 & 0.72 & 4.16 & 36.92 & 8.67 & 5.31 & 68.71 \\
\hline Java & 0.08 & 0.23 & 1.04 & 17.08 & 55.54 & 8.95 & 17.82 \\
\hline Power & 0.36 & 1.83 & 11.05 & 80.55 & 3.80 & 11.16 & 183.71 \\
\hline Erdos 02 & 0.76 & 3.62 & 21.71 & 179.75 & 44.15 & 30.42 & 358.37 \\
\hline World Air & 0.31 & 1.10 & 5.79 & 81.06 & 55.06 & 11.71 & 97.91 \\
\hline Oregon & 2.32 & 9.62 & 56.96 & 525.76 & 573.47 & 157.60 & 936.84 \\
\hline PGP & 2.42 & 9.12 & 51.31 & 603.75 & 35.74 & 57.32 & 862.56 \\
\hline Spam & 0.99 & 2.33 & 10.33 & 318.16 & 199.83 & 42.80 & 171.68 \\
\hline $\begin{array}{l}\text { Indochina } \\
\text { 2004 }\end{array}$ & 2.48 & 10.04 & 59.16 & 1086.26 & 91.95 & 74.61 & 1003.82 \\
\hline
\end{tabular}

Table 3. Time achieved by LinkPred on different prediction tasks. Column $n$ contains the number of nodes in the network, whereas $m$ shows the number of edges.

\begin{tabular}{|c|c|c|c|c|c|}
\hline Network & $n$ & $m$ & Task & Hardware & Time (sec.) \\
\hline Brightkite & 58,228 & 214,078 & $\begin{array}{l}\text { Compute ROC using } 10 \% \text { removed } \\
\text { edges for ADA. }\end{array}$ & $\begin{array}{l}1 \text { node, } 6 \text { cores (Core i7- } \\
8750 \mathrm{H})\end{array}$ & 32.92 \\
\hline Yahoo IM & 100,001 & 587,964 & Find the top $10^{4}$ edges using RAL. & $\begin{array}{l}1 \text { node, } 1 \text { core (Core i7- } \\
8750 \mathrm{H})\end{array}$ & 6.70 \\
\hline Twitter & 404,719 & 713,319 & Find the top $10^{5}$ edges using RAL. & $\begin{array}{l}1 \text { node, } 1 \text { core (Core i7- } \\
8750 \mathrm{H})\end{array}$ & 16.93 \\
\hline Youtube & $1,134,890$ & $2,987,624$ & Find the top $10^{5}$ edges using CNE. & $\begin{array}{l}1 \text { node, } 6 \text { cores (Core i7- } \\
8750 \mathrm{H})\end{array}$ & 79,41 \\
\hline CA Roads & $1,965,206$ & $2,766,607$ & Find the top $10^{5}$ edges using CNE. & $\begin{array}{l}1 \text { node, } 6 \text { cores (Core i7- } \\
8750 \mathrm{H})\end{array}$ & 7.08 \\
\hline Wiki Talks & $2,394,385$ & $4,659,565$ & Find the top $10^{5}$ edges using CNE. & $\begin{array}{l}1 \text { node, } 6 \text { cores (Core i7- } \\
8750 \mathrm{H})\end{array}$ & 470.04 \\
\hline Internet & 124,651 & 193,620 & $\begin{array}{l}\text { Compute top-precision using } 10 \% \\
\text { removed edges for eight algorithms. }\end{array}$ & $\begin{array}{l}8 \text { nodes, } 16 \text { cores in each } \\
\text { node (Xeon E5-2650) }\end{array}$ & 3.73 \\
\hline Amazon & 334,863 & 925,872 & $\begin{array}{l}\text { Compute top-precision using } 10 \% \\
\text { removed edges for eight algorithms. }\end{array}$ & $\begin{array}{l}8 \text { nodes, } 16 \text { cores in each } \\
\text { node (Xeon E5-2650) }\end{array}$ & 24.17 \\
\hline
\end{tabular}

\section{CONCLUSION AND FUTURE WORK}

LinkPred is a distributed and parallel library for link prediction in complex networks. It contains the implementation of the most important link prediction algorithms found in the literature. The library is designed not only to achieve high performance but also to be easy-to-use and extensible. The experiments show that the library can handle very large networks with up to millions of nodes and edges and is one to two orders of magnitude faster than existing Python and R packages. LinkPrted components interact through clearly defined and easy interfaces, allowing users to plug their own components into the library by implementing these interfaces. In particular, users can integrate their own link prediction algorithms and performance measures seamlessly into the library. This makes LinkPred an ideal tool for practitioners 
as well as researchers in link prediction.

The library can be improved and extended in several ways, such as adding R and Octave/Matlab bindings. Another possibility for improvement is implementing further graph embedding algorithms, particularly those based on deep neural networks. Also important is handling dynamic (time-evolving) networks. Finally, sampling-based methods such as SBM and FBM, although producing good results, are only usable with small networks because they are computationally intensive. Distributed implementations of these algorithms will allow using them in practical situations on large networks.

\section{ACKNOWLEDGMENTS}

This research work is supported by the Research Center, CCIS, King Saud University, Riyadh, Saudi Arabia. Part of the computational experiments reported in this work were conducted on the SANAM supercomputer at King Abdulaziz City for Science and Technology (hpc.kacst . edu . sa).

\section{A APPENDIX: DATA}

Table 4 below contains the description of all networks used in the experimental evaluation section.

Table 4. Description of the networks used in the experimental analysis. Columns $n$ and $m$ represent the number of nodes and edges in the network, respectively.

\begin{tabular}{|c|c|c|c|}
\hline Network & Description & $n$ & $m$ \\
\hline $\begin{array}{l}\text { Amazon(Yang } \\
\text { Leskovec, 2015) }\end{array}$ & $\begin{array}{l}\text { Amazon product co-purchasing network. An edge indicates that two } \\
\text { products have been co-purchased. Data available at https://snap. } \\
\text { stanford.edu/data/com-Amazon. html. }\end{array}$ & 334,863 & 925,872 \\
\hline $\begin{array}{l}\text { Brightkite(Cho et al., } \\
\text { 2011) }\end{array}$ & $\begin{array}{l}\text { Friendship network on the social platform Brightkite. The } \\
\text { data is available at https://snap. stanford.edu/data/ } \\
\text { loc-Brightkite.html. }\end{array}$ & 58,228 & 214,0787 \\
\hline $\begin{array}{l}\text { CA Roads(Leskovec et al., } \\
\text { 2009) }\end{array}$ & $\begin{array}{l}\text { California road network. Data available at https://snap. } \\
\text { stanford.edu/data/roadNet-CA.html. }\end{array}$ & $1,965,206$ & $2,766,607$ \\
\hline $\begin{array}{l}\text { Diseasome (Goh et al., } \\
\text { 2007) }\end{array}$ & $\begin{array}{l}\text { A network of genes' disorders and disease linked by known disor- } \\
\text { der-gene associations. The data is available at http://gephi.org/ } \\
\text { datasets/diseasome.gexf.zip. }\end{array}$ & 1,419 & 2,738 \\
\hline $\begin{array}{l}\text { Email (Guimerà et al., } \\
\text { 2003) }\end{array}$ & $\begin{array}{l}\text { The symmetrized network of email communication at the University } \\
\text { Rovira i Virgili (Tarragona, Spain). The nodes represent users, and } \\
\text { edges indicate an email communication took place between the two uses. } \\
\text { The dataset is available at http:// deim. urv. cat/ a lexandre. } \\
\text { arenas / data/welcome. htm. }\end{array}$ & 1,133 & 5,451 \\
\hline Erdos 02 & $\begin{array}{l}\text { The } 2002 \text { version of Erdös' co-authorship network. The net- } \\
\text { work is available at http://vlado. fmf.uni-lj.si/pub/ } \\
\text { networks/data/Erdos/Erdos02. net. }\end{array}$ & 6,927 & 11,850 \\
\hline $\begin{array}{l}\text { Indochina 2004(Boldi and } \\
\text { Vigna, 2004; Boldi et al., } \\
2011 \text { ) }\end{array}$ & $\begin{array}{l}\text { A WWW network available at http://networkrepository. } \\
\text { com/web_indochina_2004.php. }\end{array}$ & 11,358 & 47,606 \\
\hline $\begin{array}{l}\text { Internet(Batagelj and } \mathrm{Mr}- \\
\text { var, 2006) }\end{array}$ & $\begin{array}{l}\text { Network of Internet routers. The network is available at https:// } \\
\text { sparse.tamu.edu/Pajek/internet. }\end{array}$ & 124,651 & 193,620 \\
\hline Java & $\begin{array}{l}\text { The symmetrized version of a network where nodes represent Java } \\
\text { classes and edges represent compile-time dependencies between two } \\
\text { classes. The dataset can be found at http://vlado. fmf.uni-lj. } \\
\text { si/pub/networks/data/GD/GD. htm. }\end{array}$ & 1,538 & 7,817 \\
\hline $\begin{array}{l}\text { Oregon(Leskovec et al., } \\
\text { 2005) }\end{array}$ & $\begin{array}{l}\text { Autonomous Systems (AS) peering network inferred from Oregon route- } \\
\text { views on May 26, 2001.The data is available at https://snap. } \\
\text { stanford.edu/data/oregon1_010526.txt.gz. }\end{array}$ & 11,174 & 23,409 \\
\hline PGP (Boguñá et al., 2004) & $\begin{array}{l}\text { A social network of users using Pretty Good Privacy (PGP) al- } \\
\text { gorithm. The network is available at http://deim.urv. cat/ } \\
\sim \text { alexandre.arenas/data/welcome.htm. }\end{array}$ & 10,680 & 24,316 \\
\hline $\begin{array}{l}\text { Political Blogs (Adamic } \\
\text { and Glance, 2005) }\end{array}$ & $\begin{array}{l}\text { A network of hyperlinks among political web blogs. The data is available } \\
\text { at http: // networkrepository. com/web-polblogs.php. }\end{array}$ & 643 & 2,280 \\
\hline
\end{tabular}




\begin{tabular}{|c|c|c|c|}
\hline $\begin{array}{l}\text { PowerWatts and Strogatz } \\
\text { (1998) }\end{array}$ & $\begin{array}{l}\text { The Western States Power Grid of the United States. Data available at } \\
\text { http://www-personal.umich.edu/ mejn/netdata/. }\end{array}$ & 41 & 6,594 \\
\hline Spam(Castillo et al., 2008) & $\begin{array}{l}\text { A WWW network available at http://networkrepository. } \\
\text { com/web-spam.php. }\end{array}$ & 4,767 & 37,375 \\
\hline $\begin{array}{l}\text { Twitter (Gleich and Rossi, } \\
\text { 2014) }\end{array}$ & $\begin{array}{l}\text { A Twitter network of follow relationship. Data available at http:// } \\
\text { networkrepository.com/soc-twitter-follows.php. }\end{array}$ & 404,719 & 713,319 \\
\hline $\begin{array}{l}\text { Web Edu(Gleich et al., } \\
\text { 2004) }\end{array}$ & $\begin{array}{l}\text { A WWW network available at http://networkrepository. } \\
\text { com/web-edu.php. }\end{array}$ & 3,031 & 6,474 \\
\hline $\begin{array}{l}\text { Wiki Talks (Leskovec et al., } \\
\text { 2010b,a) }\end{array}$ & $\begin{array}{l}\text { A symmetrized version of the Wikipedia talk network. A node represents } \\
\text { a user, and an edge indicates that one user edited the talk age of another } \\
\text { user. Data available at https://snap. stanford. edu/data/ } \\
\text { wiki-Talk.html. }\end{array}$ & $2,394,385$ & $4,659,565$ \\
\hline $\begin{array}{l}\text { World Transport (Guimerà } \\
\text { et al., 2005) }\end{array}$ & $\begin{array}{l}\text { A worldwide airport network. Nodes represent cities, and edges } \\
\text { indicate a flight connecting two cities. The data is available at } \\
\text { http://seeslab.info/media/filer_public/63/97/ } \\
63979 \mathrm{ddc}-\mathrm{a} 625-42 \mathrm{f} 9-9 \mathrm{~d} 3 \mathrm{~d}-8 \mathrm{fdb} 4 \mathrm{~d} 6 \mathrm{ce} 0 \mathrm{~b} 0 / \text { airports. } \\
\text { zip. }\end{array}$ & 3,618 & 14,142 \\
\hline $\begin{array}{l}\text { Yahoo IM (Yahoo! Web- } \\
\text { scope, 2008) }\end{array}$ & $\begin{array}{l}\text { Network of sample Yahoo! Messenger communication events. The } \\
\text { data is available at https://webscope. sandbox.yahoo.com/ } \\
\text { catalog.php?datatype=g. }\end{array}$ & 100,001 & 587,964 \\
\hline $\begin{array}{l}\text { Youtube (Yang et al., } \\
\text { 2015a) }\end{array}$ & $\begin{array}{l}\text { A Youtube friendship network. Data available at https://snap. } \\
\text { stanford.edu/data/com-Youtube.html. }\end{array}$ & $1,134,890$ & $2,987,624$ \\
\hline $\begin{array}{l}\text { Zakary's Karate Club } \\
\text { (Zachary, 1977) }\end{array}$ & $\begin{array}{l}\text { A friendship network among members of a karate club at an Amer- } \\
\text { ican university. The data was collected in the } 1970 \text { s by Wayne } \\
\text { Zachary and is available at http://konect.cc/networks/ } \\
\text { ucidata-zachary }\end{array}$ & 34 & 78 \\
\hline
\end{tabular}

\section{REFERENCES}

Adamic, L. A. and Adar, E. (2003). Friends and neighbors on the web. Social networks, 25(3):211-230.

Adamic, L. A. and Glance, N. (2005). The political blogosphere and the 2004 us election: divided they blog. In Proceedings of the 3rd international workshop on Link discovery, pages 36-43. ACM.

Ahmed, A., Shervashidze, N., Narayanamurthy, S., Josifovski, V., and Smola, A. J. (2013). Distributed large-scale natural graph factorization. In Proceedings of the 22nd International Conference on World Wide Web, WWW'13, pages 37-48, New York, NY, USA. Association for Computing Machinery.

Al Hasan, M., Chaoji, V., Salem, S., and Zaki, M. (2006). Link prediction using supervised learning. In SDM'06: Workshop on Link Analysis, Counter-terrorism and Security.

Albert, R. and Barabási, A.-L. (2002). Statistical mechanics of complex networks. Reviews of modern physics, 74(1):47-97.

Alharbi, R., Benhidour, H., and Kerrache, S. (2016). Link prediction in complex networks based on a hidden variables model. In 2016 UKSim-AMSS 18th International Conference on Computer Modelling and Simulation (UKSim), pages 119-124.

Batagelj, V. and Mrvar, A. (2006). Pajek datasets. http://vlado.fmf.uni-lj.si/pub/networks/data.

Beigi, G., Tang, J., and Liu, H. (2020). Social science-guided feature engineering: A novel approach to signed link analysis. ACM Trans. Intell. Syst. Technol., 11(1).

Belkin, M. and Niyogi, P. (2001). Laplacian eigenmaps and spectral techniques for embedding and clustering. In Dietterich, T. G., Becker, S., and Ghahramani, Z., editors, NIPS, pages 585-591. MIT Press.

Bhagat, S., Cormode, G., and Muthukrishnan, S. (2011). Node classification in social networks. In Aggarwal, C. C., editor, Social Network Data Analytics, pages 115-148. Springer.

Boguñá, M., Pastor-Satorras, R., Díaz-Guilera, A., and Arenas, A. (2004). Models of social networks based on social distance attachment. Physical review E, 70(5):056122.

Bojanowski, M. and Chrol, B. (2019). linkprediction: Link Prediction Methods. R package version 1.0-1.

Boldi, P., Rosa, M., Santini, M., and Vigna, S. (2011). Layered label propagation: A multiresolution coordinate-free ordering for compressing social networks. In Srinivasan, S., Ramamritham, K., Kumar, A., Ravindra, M. P., Bertino, E., and Kumar, R., editors, Proceedings of the 20th international conference on World Wide Web, pages 587-596. ACM Press.

Boldi, P. and Vigna, S. (2004). The WebGraph framework I: Compression techniques. In Proc. of the Thirteenth International World Wide Web Conference (WWW 2004), pages 595-601, Manhattan, USA. ACM Press.

Bonald, T., de Lara, N., Lutz, Q., and Charpentier, B. (2020). Scikit-network: Graph analysis in python. Journal of Machine Learning Research, 21(185):1-6.

Cannistraci, C. V., Alanis-Lobato, G., and Ravasi, T. (2013). From link-prediction in brain connectomes and protein interactomes to the local-community-paradigm in complex networks. Scientific reports, 3.

Cao, S., Lu, W., and Xu, Q. (2016). Deep neural networks for learning graph representations. In AAAI Conference on Artificial Intelligence.

Castillo, C., Chellapilla, K., and Denoyer, L. (2008). Web spam challenge 2008. In Proceedings of the 4th International Workshop on Adversarial Information Retrieval on the Web (AIRWeb). 
Cho, E., Myers, S. A., and Leskovec, J. (2011). Friendship and mobility: User movement in location-based social networks. In Proceedings of the 17th ACM SIGKDD International Conference on Knowledge Discovery and Data Mining, KDD '11, pages 1082-1090, New York, NY, USA. ACM.

Clauset, A., Moore, C., and Newman, M. E. (2008). Hierarchical structure and the prediction of missing links in networks. Nature, 453(7191):98-101.

Curtin, R. R., Cline, J. R., Slagle, N. P., March, W. B., Ram, P., Mehta, N. A., and Gray, A. G. (2013). Mlpack: A scalable c++ machine learning library. J. Mach. Learn. Res., 14(1):801-805.

Daminelli, S., Thomas, J. M., Durán, C., and Cannistraci, C. V. (2015). Common neighbours and the local-community-paradigm for topological link prediction in bipartite networks. New Journal of Physics, 17(11):113037.

Davis, J. and Goadrich, M. (2006). The Relationship Between Precision-Recall and ROC Curves. In Proceedings of the 23rd International Conference on Machine Learning, ICML '06, pages 233-240, New York, NY, USA. ACM.

Donnat, C., Zitnik, M., Hallac, D., and Leskovec, J. (2018). Learning structural node embeddings via diffusion wavelets. In International ACM Conference on Knowledge Discovery and Data Mining (KDD), volume 24.

Gleich, D., Zhukov, L., and Berkhin, P. (2004). Fast parallel pagerank: A linear system approach. Yahoo! Research Technical Report YRL-2004-038, 13:22.

Gleich, D. F. and Rossi, R. A. (2014). A dynamical system for pagerank with time-dependent teleportation. Internet Mathematics, 10(1-2):188-217.

Goh, K.-I., Cusick, M. E., Valle, D., Childs, B., Vidal, M., and Barabási, A.-L. (2007). The human disease network. Proceedings of the National Academy of Sciences, 104(21):8685-8690.

Goyal, P. and Ferrara, E. (2018a). Gem: A python package for graph embedding methods. Journal of open source software, 3(29):876.

Goyal, P. and Ferrara, E. (2018b). Graph embedding techniques, applications, and performance: A survey. Knowledge-Based Systems.

Goyal, P. and Ferrara, E. (2018c). Graph embedding techniques, applications, and performance: A survey. Knowledge-Based Systems, 151:78- 94

Grover, A. and Leskovec, J. (2016). Node2vec: Scalable feature learning for networks. In Proceedings of the 22nd ACM SIGKDD International Conference on Knowledge Discovery and Data Mining, KDD '16, pages 855-864, New York, NY, USA. Association for Computing Machinery.

Guimerà, R., Danon, L., Díaz-Guilera, A., Giralt, F., and Arenas, A. (2003). Self-similar community structure in a network of human interactions. Phys. Rev. E, 68:065103.

Guimerà, R., Mossa, S., Turtschi, A., and Amaral, L. A. N. (2005). The worldwide air transportation network: Anomalous centrality, community structure, and cities' global roles. Proceedings of the National Academy of Sciences of the United States of America, 102(22):7794-9.

Guimerà, R. and Sales-Pardo, M. (2009). Missing and spurious interactions and the reconstruction of complex networks. Proceedings of the National Academy of Sciences, 106(52):22073-22078.

Hagberg, A., Schult, D., and Swart, P. (2019). Networkx: Software for complex networks. https: // networkx . github .io. Accessed: 2021-03-06.

Jaccard, P. (1901). Étude comparative de la distribution florale dans une portion des alpes et des jura. Bull Soc Vaudoise Sci Nat, 37:547-579.

Jeh, G. and Widom, J. (2002). Simrank: A measure of structural-context similarity. In Proceedings of the Eighth ACM SIGKDD International Conference on Knowledge Discovery and Data Mining, KDD '02, page 538-543, New York, NY, USA. Association for Computing Machinery.

Katz, L. (1953). A new status index derived from sociometric analysis. Psychometrika, 18(1):39-43.

Kazemi, S. M. and Poole, D. (2018). Simple embedding for link prediction in knowledge graphs. In Bengio, S., Wallach, H., Larochelle, H., Grauman, K., Cesa-Bianchi, N., and Garnett, R., editors, Advances in Neural Information Processing Systems, volume 31, pages 4284-4295. Curran Associates, Inc.

Kerrache, S., Alharbi, R., and Benhidour, H. (2020). A scalable similarity-popularity link prediction method. Scientific reports, 10(1):6394-6394.

Koren, Y., Bell, R., and Volinsky, C. (2009). Matrix factorization techniques for recommender systems. Computer, 42(8):30-37.

Leskovec, J., Huttenlocher, D., and Kleinberg, J. (2010a). Predicting positive and negative links in online social networks. In Proceedings of the 19th international conference on world wide web, WWW '10, pages 641-650. ACM.

Leskovec, J., Huttenlocher, D., and Kleinberg, J. (2010b). Signed networks in social media. In Proceedings of the SIGCHI Conference on human factors in computing systems, CHI '10, pages 1361-1370. ACM.

Leskovec, J., Kleinberg, J., and Faloutsos, C. (2005). Graphs over time: densification laws, shrinking diameters and possible explanations. In Proceedings of the eleventh ACM SIGKDD international conference on Knowledge discovery in data mining, KDD '05, pages 177-187, New York, NY, USA. ACM.

Leskovec, J., Lang, K. J., Dasgupta, A., and Mahoney, M. W. (2009). Community structure in large networks: Natural cluster sizes and the absence of large well-defined clusters. Internet mathematics, 6(1):29-123.

Leskovec, J. and Sosič, R. (2016). Snap: A general-purpose network analysis and graph-mining library. ACM Transactions on Intelligent Systems and Technology (TIST), 8(1):1.

Liben-Nowell, D. and Kleinberg, J. (2007). The link-prediction problem for social networks. Journal of the American society for information science and technology, 58(7):1019-1031.

Lichtenwalter, R. N., Lussier, J. T., and Chawla, N. V. (2010). New perspectives and methods in link prediction. In Proceedings of the 16th ACM SIGKDD international conference on Knowledge discovery and data mining, pages 243-252. ACM.

linkpred (2020). linkpred: A python package for link prediction. https://github.com/rafguns/linkpred. Accessed: 2021-03-25.

Liu, W. and Lü, L. (2010). Link prediction based on local random walk. EPL (Europhysics Letters), 89(5):58007.

Liu, Z., He, J.-L., Kapoor, K., and Srivastava, J. (2013). Correlations between community structure and link formation in complex networks. PloS one, 8(9)

Lü, L. and Zhou, T. (2011). Link prediction in complex networks: A survey. Physica A: Statistical Mechanics and its Applications, 
390(6):1150-1170

Makarov, I., Gerasimova, O., Sulimov, P., and Zhukov, L. E. (2019). Dual network embedding for representing research interests in the link prediction problem on co-authorship networks. PeerJ. Computer science, 5:e172.

Martínez, V., Berzal, F., and Cubero, J.-C. (2016). A survey of link prediction in complex networks. ACM Comput. Surv., 49(4).

Muscoloni, A. and Cannistraci, C. V. (2017). Local-ring network automata and the impact of hyperbolic geometry in complex network link-prediction. Preprint at http://arXiv:1707.09496 [physics.soc-ph].

Neo4J (2019). Neo4j graph platform. https : / / neo 4 j . com. Accessed: 2021-03-06.

Newman, M. E. J. (2001). Clustering and preferential attachment in growing networks. Phys. Rev. E, 64.

Ou, M., Cui, P., Pei, J., Zhang, Z., and Zhu, W. (2016). Asymmetric transitivity preserving graph embedding. In Proceedings of the 22nd ACM SIGKDD International Conference on Knowledge Discovery and Data Mining, KDD '16, pages 1105-1114, New York, NY, USA. Association for Computing Machinery.

Papadimitriou, A., Symeonidis, P., and Manolopoulos, Y. (2012). Fast and accurate link prediction in social networking systems. Journal of Systems and Software, 85(9):2119-2132.

Papadopoulos, F., Kitsak, M., Serrano, M. Á., Boguná, M., and Krioukov, D. (2012). Popularity versus similarity in growing networks. Nature, 489(7417):537-540.

Papadopoulos, F., Psomas, C., and Krioukov, D. (2015). Network mapping by replaying hyperbolic growth. IEEE/ACM Transactions on Networking (TON), 23(1):198-211.

Perozzi, B., Al-Rfou, R., and Skiena, S. (2014). Deepwalk: Online learning of social representations. In Proceedings of the 20th ACM SIGKDD International Conference on Knowledge Discovery and Data Mining, KDD '14, pages 701-710, New York, NY, USA. Association for Computing Machinery.

Ravasz, E., Somera, A. L., Mongru, D. A., Oltvai, Z. N., and Barabási, A.-L. (2002). Hierarchical organization of modularity in metabolic networks. science, 297(5586):1551-1555.

Roweis, S. T. and Saul, L. K. (2000). Nonlinear dimensionality reduction by locally linear embedding. Science, 290(5500):23232326.

Sajadmanesh, S., Bazargani, S., Zhang, J., and Rabiee, H. R. (2019). Continuous-time relationship prediction in dynamic heterogeneous information networks. ACM Trans. Knowl. Discov. Data, 13(4).

Tang, J., Aggarwal, C. C., and Liu, H. (2016a). Node classification in signed social networks. In Venkatasubramanian, S. C. and Jr., W. M., editors, SDM, pages 54-62. SIAM.

Tang, J., Liu, J., Zhang, M., and Mei, Q. (2016b). Visualizing large-scale and high-dimensional data. In Bourdeau, J., Hendler, J., Nkambou, R., Horrocks, I., and Zhao, B. Y., editors, WWW, pages 287-297. ACM.

Tang, J., Qu, M., Wang, M., Zhang, M., Yan, J., and Mei, Q. (2015). LINE: Large-Scale Information Network Embedding, page 1067-1077. International World Wide Web Conferences Steering Committee, Republic and Canton of Geneva, CHE.

van der Maaten, L. and Hinton, G. (2008). Visualizing data using t-SNE. Journal of Machine Learning Research, 9:2579-2605.

Wang, C., Satuluri, V., and Parthasarathy, S. (2007). Local probabilistic models for link prediction. In Proceedings of the 2007 Seventh IEEE International Conference on Data Mining, pages 322-331. IEEE.

Wang, D., Cui, P., and Zhu, W. (2016). Structural deep network embedding. In Proceedings of the 22nd ACM SIGKDD International Conference on Knowledge Discovery and Data Mining, KDD '16, pages 1225-1234, New York, NY, USA. Association for Computing Machinery.

Watts, D. J. and Strogatz, S. H. (1998). Collective dynamics of 'small-world'networks. nature, 393(6684):440-442.

Yahoo! Webscope (2008). Dataset: ydata-ymessenger-user-communication-pattern-v1_0.

Yang, J. and Leskovec, J. (2015). Defining and evaluating network communities based on ground-truth. Knowl. Inf. Syst., 42(1):181-213.

Yang, J., Yang, J., Leskovec, J., and Leskovec, J. (2015a). Defining and evaluating network communities based on ground-truth Knowledge and information systems, 42(1):181-213.

Yang, J., Yang, L., and Zhang, P. (2015b). A new link prediction algorithm based on local links. In Web-Age Information Management, pages 16-28. Springer.

Yang, Y., Lichtenwalter, R. N., and Chawla, N. V. (2015c). Evaluating link prediction methods. Knowledge and Information Systems, 45(3):751-782.

Zachary, W. W. (1977). An information flow model for conflict and fission in small groups. Journal of anthropological research, 33:452-473.

Zhang, R., Mensah, S., Kong, F., Hu, Z., Mao, Y., and Liu, X. (2020). Pairwise link prediction model for out of vocabulary knowledge base entities. ACM Trans. Inf. Syst., 38(4).

Zhu, B. and Xia, Y. (2015). An information-theoretic model for link prediction in complex networks. Scientific reports, 5. 